\title{
Determination of an additive time- and space-dependent coefficient in the heat equation
}

\author{
M.J. Huntul ${ }^{1,2}$, D. Lesnic ${ }^{1}$ and B.T. Johansson \\ ${ }^{1}$ Department of Applied Mathematics, University of Leeds, Leeds LS2 9JT, UK \\ ${ }^{2}$ Department of Mathematics, College of Science, Jazan University, Jazan, Saudia Arabia
}

E-mails: mmmjmh@leeds.ac.uk (M.J.Huntul), amt5ld@maths.leeds.ac.uk (D. Lesnic),

\begin{abstract}
In this paper, we consider the inverse problem of determining the additive time- and space-dependent perfusion coefficient in the parabolic heat equation. Using initial and boundary conditions as well as temperature measurements at an interior space location and at the final time ensure that the inverse problem has a unique solution. However, the problem is still ill-posed since small errors in the input data cause large errors in the output solution. For the numerical discretization, the finite difference method combined with a regularized nonlinear optimization are employed. Numerical results are illustrated in order to show the efficiency of the adopted computational methods.
\end{abstract}

Keywords: Inverse problem; finite-difference method; nonlinear optimization; regularization; bioheat equation; perfusion coefficient.

\section{Introduction}

Inverse problems for the parabolic heat equation consisting of determining the unknown radiative/absorbtion/perfusion coefficient depending on both time and space have recently received some attention, $[1,4,5,11]$. The knowledge of this physical property is very important in understanding the heat transfer in biological tissues, [13]. Its direct measurement is not available in the general case when it depends on both space and time. However, it can be inferred by inverse methods based on the measurement of the interior temperature, as considered in [14]. On the other hand, this formulation means that infinitely many intrusive measurements of temperature with thermocouples embedded inside the material are necessary at all space points and for all times. A possible alternative to this general inverse modelling is to restrict the generality of the coefficient by seeking it as a sum of a function dependent of time and one dependent of space. This additive class in which the admissible coefficient is sought allows to formulate an inverse problem for which a single measurement of the temperature in time at a fixed space point and one in space at a fixed instant are sufficient to ensure that the identification is possible. A similar approach has previously been investigated in related problems concerned with the identification of an additive heat source, $[6,7]$. However, the inverse heat source problem is linear whilst the coefficient identification problem investigated in this paper is nonlinear and this significantly complicates its study.

The paper is structured as follows: In Section 2, the mathematical formulation of the inverse problem is given. In Section 3, the numerical solution of direct problem based 
on finite difference method (FDM) with Crank-Nicolson scheme is briefly introduced. In Section 4, the numerical approach to solve the inverse problem based on a minimization algorithm is presented. Numerical results are presented and discussed in Section 5. Finally, conclusions are presented in Section 6.

\section{Mathematical formulation}

Fix the parameters $L>0$ and $T>0$ representing the length of a finite slab and the time duration, respectively. Denote by $Q_{T}=\{(x, t) \mid 0<t<T, 0<x<L\}$ the solution domain. We consider the parabolic heat equation

$$
\frac{\partial u}{\partial t}(x, t)=\frac{\partial^{2} u}{\partial x^{2}}(x, t)+(f(t)+g(x)) u(x, t), \quad(x, t) \in Q_{T},
$$

where $f(t)$ and $g(x)$ are coefficient functions to be identified together with the temperature $u(x, t)$. Equation (1) has to be solved subject to the initial condition

$$
u(x, 0)=\phi(x), \quad 0 \leq x \leq L,
$$

the homogeneous Neumann boundary conditions

$$
\frac{\partial u}{\partial x}(0, t)=\frac{\partial u}{\partial x}(L, t)=0, \quad 0 \leq t \leq T,
$$

and the additional temperature measurements

$$
u\left(X_{0}, t\right)=\beta(t), \quad 0 \leq t \leq T,
$$

at a fixed space location $0<X_{0}<L$, and

$$
u(x, T)=\psi(x), \quad 0 \leq x \leq L,
$$

at the final time $t=T$. The conditions (3) express that the ends $\{0, L\}$ of the finite slab $(0, L)$ are insulated. In order to avoid non-uniqueness reproduced by the trivial identity $f(t)+g(x)=f(t)+c+g(x)-c$, where $c$ is an arbitrary non-zero constant, we take a fixing condition, say at $x=X_{1}$ fixed in $(0, L)$, assuming that

$$
g\left(X_{1}\right)=\alpha
$$

is given. Alternatively, one could have a fixing condition on $f$ instead of (6). In the above equations, the functions $\phi, \beta, \psi$ and the constant $\alpha$ are given, whilst the triplet of functions $f(t), g(x)$ and $u(x, t)$ are unknown. Further, assume that the conditions (2)-(5) are compatible, i.e.

$$
\phi^{\prime}(0)=\phi^{\prime}(L)=\psi^{\prime}(0)=\psi^{\prime}(L)=0, \quad \beta(0)=\phi\left(X_{0}\right), \quad \beta(T)=\psi\left(X_{0}\right) .
$$

The existence and uniqueness of a classical solution to the inverse problem (1)-(7) were established in [11] and, for convenience, the uniqueness theorem is stated correctly and proved in Appendix A. We note that we do not take $X_{0}$ to be a boundary point because the proof in [11] starts by applying equation (1) and using (4) at $x=X_{0} \in(0, L)$. Without going in too much detail, for illustration it is useful to state the unique solvability of the 
inverse problem (1)-(7) in a particular case, as follows.

Proposition 1. Suppose

$$
0<\phi \in C^{4}([0, L]), \quad 0<\psi \in C^{4}([0, L]), \quad 0<\beta \in C^{1}([0, T])
$$

and assume that

$$
\psi(x)=c \phi(x), x \in[0, L]
$$

where $c=\beta(T) / \beta(0)$. Then the inverse problem (1)-(7) has a unique solution $(u, f, g) \in$ $\left(C^{2}\left(Q_{T}\right) \cap C^{1}\left(\overline{Q_{T}}\right)\right) \times C^{1}([0, T]) \times C^{1}([0, L])$ which is explicitly given by

$$
\begin{gathered}
u(x, t)=\frac{\beta(t)}{\beta(0)} \phi(x), \quad(x, t) \in \overline{Q_{T}}, \\
f(t)=\frac{\beta^{\prime}(t)}{\beta(t)}-\alpha-\frac{\phi^{\prime \prime}\left(X_{1}\right)}{\phi\left(X_{1}\right)}, \quad t \in[0, T], \\
g(x)=-\frac{\phi^{\prime \prime}(x)}{\phi(x)}+\alpha+\frac{\phi^{\prime \prime}\left(X_{1}\right)}{\phi\left(X_{1}\right)}, \quad x \in[0, L] .
\end{gathered}
$$

Proof. We proceed as in [11] and remark that from (1)-(3) using the maximum principle for the parabolic heat equation we have that $u>0$ in $\overline{Q_{T}}$ and let us put

$$
u=e^{v}
$$

Then equations (1)-(5) become

$$
\begin{gathered}
v_{t}(x, t)=v_{x x}(x, t)+v_{x}^{2}(x, t)+f(t)+g(x), \quad(x, t) \in Q_{T}, \\
v(x, 0)=\ln (\phi(x))=: \Phi(x), \quad x \in[0, L], \\
v_{x}(0, t)=v_{x}(L, t)=0, \quad t \in[0, T], \\
v(x, T)=\ln (\psi(x))=: \Psi(x), \quad x \in[0, L], \\
v\left(X_{0}, t\right)=\ln (\beta(t))=: \beta_{1}(t), \quad t \in[0, T] .
\end{gathered}
$$

From (9), (15) and (17) one can see that

$$
\Psi(x)=\Phi(x)+\ln (c), \quad x \in[0, L] .
$$

Differentiating equation (14) with respect to $x$ we get

$$
v_{t x}(x, t)=v_{x x x}(x, t)+2 v_{x}(x, t) v_{x x}(x, t)+g^{\prime}(x), \quad(x, t) \in Q_{T} .
$$


Now differentiating (20) with respect to $t$ and using (17) we obtain, [11],

$$
\begin{aligned}
& w_{t}(x, t)=w_{x x}(x, t)+2\left(\int_{T}^{t} w(x, \tau) d \tau+\Psi^{\prime}(x)\right) w_{x} \\
& +2\left(\int_{T}^{t} w_{x}(x, \tau) d \tau+\Psi^{\prime \prime}(x)\right) w(x, t), \quad(x, t) \in Q_{T},
\end{aligned}
$$

where

$$
w(x, t)=v_{t x}(x, t) .
$$

Also, from (15)-(17), (19) and (20) one can derive that

$$
\begin{gathered}
w(0, t)=w(L, t)=0, \quad t \in[0, T], \\
w(x, T)-w(x, 0)=v_{x x x}(x, T)+2 v_{x}(x, T) v_{x x}(x, T)+g^{\prime}(x)-v_{x x x}(x, 0) \\
-2 v_{x}(x, 0) v_{x x}(x, 0)-g^{\prime}(x)=\Psi^{\prime \prime \prime}(x)+2 \Psi^{\prime}(x) \Psi^{\prime \prime}(x)-\Phi^{\prime \prime \prime}(x)-2 \Phi^{\prime}(x) \Phi^{\prime \prime}(x)=0, \\
x \in[0, L] .
\end{gathered}
$$

Then, one can observe that the initial boundary value problem for the parabolic equation (21) has only the trivial solution $w \equiv 0$. Hence, from (22) we obtain

$$
v(x, t)=a(t)+b(x) .
$$

Then, (14)-(19) give

$$
\begin{gathered}
a^{\prime}(t)=b^{\prime \prime}(x)+b^{\prime 2}(x)+f(t)+g(x), \quad(x, t) \in Q_{T}, \\
\Phi(x)=\ln (\phi(x))=a(0)+b(x), \quad x \in[0, L], \\
b^{\prime}(0)=b^{\prime}(L)=0, \\
\Phi(x)+\ln (c)=\Psi(x)=\ln (\psi(x))=a(T)+b(x), \quad x \in[0, L], \\
\beta_{1}(t)=\ln (\beta(t))=a(t)+b\left(X_{0}\right), \quad t \in[0, T] .
\end{gathered}
$$

From (26), we immediately obtain that

$$
f(t)=a^{\prime}(t)-C, \quad g(x)=-b^{\prime 2}(x)-b^{\prime \prime}(x)+C,
$$

where $C$ is some arbitrary constant. From (27), (29) and (30) we obtain

$$
b(x)=\ln (\phi(x))+\text { const. }, \quad a(t)=\ln (\beta(t))+\text { const } .
$$

Then, using (13), (25) and (32) we obtain that

$$
u(x, t)=C_{1} \beta(t) \phi(x)
$$


for some constant $C_{1}$,

$$
f(t)=\frac{\beta^{\prime}(t)}{\beta}-C, \quad g(x)=-\frac{\phi^{\prime \prime}(x)}{\phi(x)}+C .
$$

Imposing (6) we obtain $C=\frac{\phi^{\prime \prime}\left(X_{1}\right)}{\phi\left(X_{1}\right)}+\alpha$ from which (11) and (12) follows. Finally, from (4), (7) and (33) we obtain that $C_{1}=1 / \beta(0)$ from which (10) folows. This completes the proof of the proposition.

This proposition is useful because it indicates how to construct analytical solutions of the inverse problem (1)-(7) for which numerical methods can be assessed, see later on Examples 1 and 2 in Section 5.

Although the inverse problem (1)-(7) is uniquely solvable it is still ill-posed since small errors in the input measured data (4) and (5) cause large error in the output solution for $f$ and $g$. Therefore, in the numerical computation the main focus is on the development of stable optimization algorithms, [10], as will be described in Section 4. But before we do that, in the next section we briefly describe the FDM employed for discretising the direct problem.

\section{$3 \quad$ Numerical solution of direct problem}

In this section, we consider the direct initial boundary value problem given by equations (1)-(3) when $f$ and $g$ are given. We use the finite-difference method (FDM) with a Crank-Nicholson scheme, [12], which is unconditionally stable and second-order accurate in space and time. The discrete form of the direct problem is as follows. We denote $u\left(x_{i}, t_{j}\right)=u_{i, j}, f\left(t_{j}\right)=f_{j}$ and $g\left(x_{i}\right)=g_{i}$, where $x_{i}=i \Delta x, t_{j}=j \Delta t$ for $i=\overline{0, M}, j=\overline{0, N}$, and $\Delta x=\frac{L}{M}, \Delta t=\frac{T}{N}$.

Considering the general partial differential equation

$$
u_{t}=G\left(x, t, u, u_{x x}\right)
$$

the Crank-Nicolson method, [12], discretises (35), (2) and (3) as

$$
\begin{gathered}
\frac{u_{i, j+1}-u_{i, j}}{\Delta t}=\frac{1}{2}\left(G_{i, j}+G_{i, j+1}\right), \quad i=\overline{1,(M-1)}, j=\overline{0,(N-1)} \\
u_{i, 0}=\phi\left(x_{i}\right), \quad i=\overline{0, M}, \\
\frac{u_{1, j}-u_{-1, j}}{2(\Delta x)}=\frac{u_{M+1, j}-u_{M-1, j}}{2(\Delta x)}=0, \quad j=\overline{1, N}
\end{gathered}
$$

where

$$
\begin{array}{r}
G_{i, j}=G\left(x_{i}, t_{j}, u_{i, j}, \frac{u_{i+1, j}-2 u_{i, j}+u_{i-1, j}}{(\Delta x)^{2}}\right), \\
G_{i, j+1}=G\left(x_{i}, t_{j+1}, u_{i, j+1}, \frac{u_{i+1, j+1}-2 u_{i, j+1}+u_{i-1, j+1}}{(\Delta x)^{2}}\right) \\
i=\overline{0, M}, \quad j=\overline{0,(N-1)}
\end{array}
$$

and $u_{-1, j}$ and $u_{M+1, j}$ for $j=\overline{1, N}$ are fictitious values at points located outside the computational domain. For our problem, equation (1) can be discretised in the form of (35) as

$$
-A u_{i-1, j+1}+\left(1+B_{j+1}\right) u_{i, j+1}-A u_{i+1, j+1}=A u_{i-1, j}+\left(1-B_{j}\right) u_{i, j}+A u_{i+1, j},
$$


for $i=\overline{0, M}, j=\overline{0,(N-1)}$, where $A=\frac{(\Delta t)}{2(\Delta x)^{2}}, B_{i, j}=\frac{(\Delta t)}{(\Delta x)^{2}}-\frac{(\Delta t)}{2}\left(f_{j}+g_{i}\right)$.

At each time step $t_{j+1}$, for $j=\overline{0,(N-1)}$, using the homogeneous Neumann boundary conditions (38), the above difference equation can be reformulated as a $M \times M$ system of linear equations of the form,

$$
L \mathbf{u}_{\mathbf{j}+\mathbf{1}}=E \mathbf{u}_{\mathbf{j}}
$$

where

$$
\begin{gathered}
\mathbf{u}_{\mathbf{j}+\mathbf{1}}=\left(u_{0, j+1}, u_{1, j+1}, \ldots, u_{M, j+1}\right)^{\mathrm{T}}, \\
L=\left(\begin{array}{ccccccc}
1+B_{0, j+1} & -2 A & 0 & \ldots & 0 & 0 & 0 \\
-A & 1+B_{1, j+1} & -A & \ldots & 0 & 0 & 0 \\
\vdots & \vdots & \vdots & \ddots & \vdots & \vdots & \vdots \\
0 & 0 & 0 & \ldots & -A & 1+B_{M-1, j+1} & -A \\
0 & 0 & 0 & \ldots & 0 & -2 A & 1+B_{M, j+1}
\end{array}\right),
\end{gathered}
$$

and

$$
E=\left(\begin{array}{ccccccc}
1-B_{0, j} & 2 A & 0 & \ldots & 0 & 0 & 0 \\
A & 1-B_{1, j} & A & \ldots & 0 & 0 & 0 \\
\vdots & \vdots & \vdots & \ddots & \vdots & \vdots & \vdots \\
0 & 0 & 0 & \ldots & A & 1-B_{M-1, j} & A \\
0 & 0 & 0 & \ldots & 0 & 2 A & 1-B_{M, j}
\end{array}\right)
$$

\section{Numerical approach to solve the inverse problem}

In this section we wish to obtain simultaneously the unknown functions $f(t)$ and $g(x)$ in the inverse problem (1)-(7) reformulated as minimizing the regularized objective function

$$
\begin{aligned}
\mathbb{F}(f, g) & =\|u(x, T)-\psi(x)\|^{2}+\left\|u\left(X_{0}, t\right)-\beta(t)\right\|^{2}+\beta_{1}\|f(t)\|^{2}+\beta_{2}\|g(x)\|^{2} \\
& +\left(g\left(X_{1}\right)-\alpha\right)^{2},
\end{aligned}
$$

where $\beta_{1} \geq 0$ and $\beta_{2} \geq 0$ are regularization parameters, and the norm is usually the $L^{2}$-norm. Assuming, for convenience, that we take $X_{0} \in(0, L)$ such that there exists $i_{0} \in\{1, \ldots, M\}$ for which $X_{0}=x_{i_{0}}$, in discrete form (42) becomes

$$
\begin{aligned}
\mathbb{F}(\mathbf{f}, \mathbf{g}) & =\frac{L}{M} \sum_{\substack{i=1 \\
i \neq i_{0}}}^{M}\left[u\left(x_{i}, T\right)-\psi\left(x_{i}\right)\right]^{2}+\frac{T}{N} \sum_{j=1}^{N}\left[u\left(X_{0}, t_{j}\right)-\beta\left(t_{j}\right)\right]^{2}+\left(g\left(X_{1}\right)-\alpha\right)^{2} \\
& +\beta_{1} \sum_{j=1}^{N} f_{j}^{2}+\beta_{2} \sum_{i=1}^{M} g_{i}^{2} .
\end{aligned}
$$


The value for $i=i_{0}$ in the first sum has been excluded in order to avoid duplicating the compatibility condition (in (7)) $u\left(X_{0}, T\right)=u\left(x_{i_{0}}, T\right)=\beta(T)=\beta\left(t_{N}\right)=\psi\left(x_{i_{0}}\right)=\psi\left(X_{0}\right)$. The minimization of (43) is performed using the MATLAB toolbox routine lsqnonlin, which does not require supplying by the user the gradient of the objective function, [9]. This routine attempts to find the minimum of a sum of squares by starting from the arbitrary initial guesses $\mathbf{f}^{(0)}, \mathbf{g}^{(0)}$ for $\mathbf{f}, \mathbf{g}$, respectively. We have compiled this routine with the following parameters:

- Algorithm = Trust-Region-Reflective (TRR), [2].

- Number of variables $M=N$.

- Maximum number of iterations, (MaxIter) $=400$.

- Maximum number of objective function evaluations, (MaxFunEvals) $=10^{2} \times($ number of variables.)

- Termination tolerance on the function value, (TolFun) $=10^{-20}$.

- $\mathrm{x}$ Tolerance, $(\mathrm{xTol})=10^{-20}$.

- The lower and upper bounds, LB and UB, are $L B=-10^{3} *$ ones $(1, \operatorname{length}(\mathbf{f}, \mathbf{g}))$ and $U B=10^{3} *$ ones $(1$, length $(\mathbf{f}, \mathbf{g}))$.

The inverse problem under investigation is solved subject to both exact and noisy data which are numerically simulated as

$$
\begin{gathered}
\beta^{\epsilon 1}\left(t_{j}\right)=\beta\left(t_{j}\right)+\epsilon 1_{j}, \quad j=\overline{1, N}, \\
\psi^{\epsilon 2}\left(x_{i}\right)=\psi\left(x_{i}\right)+\epsilon 2_{i}, \quad i=\overline{1, M}, \quad i \neq i_{0},
\end{gathered}
$$

where $\epsilon 1_{j}$ and $\epsilon 2_{i}$ are random variables generated from Gaussian normal distributions with mean zero and standard deviations $\sigma 1$ and $\sigma 2$ given by

$$
\sigma 1=p \times \max _{t \in[0, T]}|\beta(t)|, \quad \sigma 2=p \times \max _{x \in[0, L]}|\psi(x)|,
$$

where $p$ represents the percentage of noise. We use the MATLAB function normrnd to generate the random variables $\underline{\epsilon 1}=\left(\epsilon 1_{j}\right)_{j=\overline{1, N}}, \underline{\epsilon 2}=\left(\epsilon 2_{i}\right)_{i=\overline{1,(M-1)}}$, as follows:

$$
\underline{\epsilon 1}=\operatorname{normrnd}(0, \sigma 1, N), \quad \underline{\epsilon 2}=\operatorname{normrnd}(0, \sigma 2, M-1) .
$$

\section{$5 \quad$ Numerical results and discussion}

In this section, we present a few examples in order to test the accuracy and stability of the numerical methods introduced in Sections 3 and 4, respectively. The root mean square errors (rmse) are used to evaluate the accuracy of the numerical results as follows:

$$
\operatorname{rmse}(f)=\sqrt{\frac{1}{N} \sum_{j=1}^{N}\left(f^{\text {numerical }}\left(t_{j}\right)-f^{\operatorname{exact}}\left(t_{j}\right)\right)^{2}},
$$




$$
\operatorname{rmse}(g)=\sqrt{\frac{1}{M} \sum_{i=1}^{M}\left(g^{\text {numerical }}\left(x_{i}\right)-g^{\text {exact }}\left(x_{i}\right)\right)^{2} .}
$$

In all examples we take, for simplicity, $T=1, L=1$ and $X_{0}=X_{1}=L / 2=0.5$. Consequently, $i_{0}=M / 2$ in (43). In all the inverse calculations we take $M=N=40$.

\section{$5.1 \quad$ Example 1}

We consider the inverse problem (1)-(7) with unknown coefficients $f(t)$ and $g(x)$ and solve this inverse problem with the input data

$$
\begin{gathered}
\phi(x)=u(x, 0)=x^{2}(x-1)^{2}+1, \\
\beta(t)=u(0.5, t)=\frac{17}{16}(1+t), \quad \psi(x)=u(x, 1)=2\left(x^{2}(x-1)^{2}+1\right), \\
\alpha=g(0.5)=\frac{16}{17} .
\end{gathered}
$$

From this data one can observe that the conditions of Proposition 1 of Section 2 are satisfied and hence the inverse problem has a unique solution given by (10)-(12) which yield

$$
\begin{gathered}
f(t)=\frac{1}{1+t}, \quad g(x)=\frac{-2+12 x-12 x^{2}}{1+x^{2}-2 x^{3}+x^{4}}, \\
u(x, t)=\left(x^{2}(x-1)^{2}+1\right)(1+t) .
\end{gathered}
$$

We take the inital guess as

$$
f^{0}(t)=1-\frac{t}{2}, \quad g^{0}(x)= \begin{cases}-2+\frac{100}{17} x, & 0 \leq x \leq 0.5 \\ \frac{66}{17}-\frac{100}{17} x, & 0.5<x \leq 1\end{cases}
$$

which are straight lines passing through $f(0)=1, f(1)=1 / 2$, and $g(0)=-2, g(0.5)=$ $16 / 17, g(1)=-2$.

Considering no noise and no regularization in the measurement data (4) and (5), respectively, the objective function (43), as a function of a number of iterations plotted in Figure 1, shows a rapid decrease to a low value of $O\left(10^{-29}\right)$ in 19 iterations. Figure 2 shows the exact and numerical solutions of the functions $f(t)$ and $g(x)$, respectively. From this figure one it can be seen that very accurate solutions are obtained.

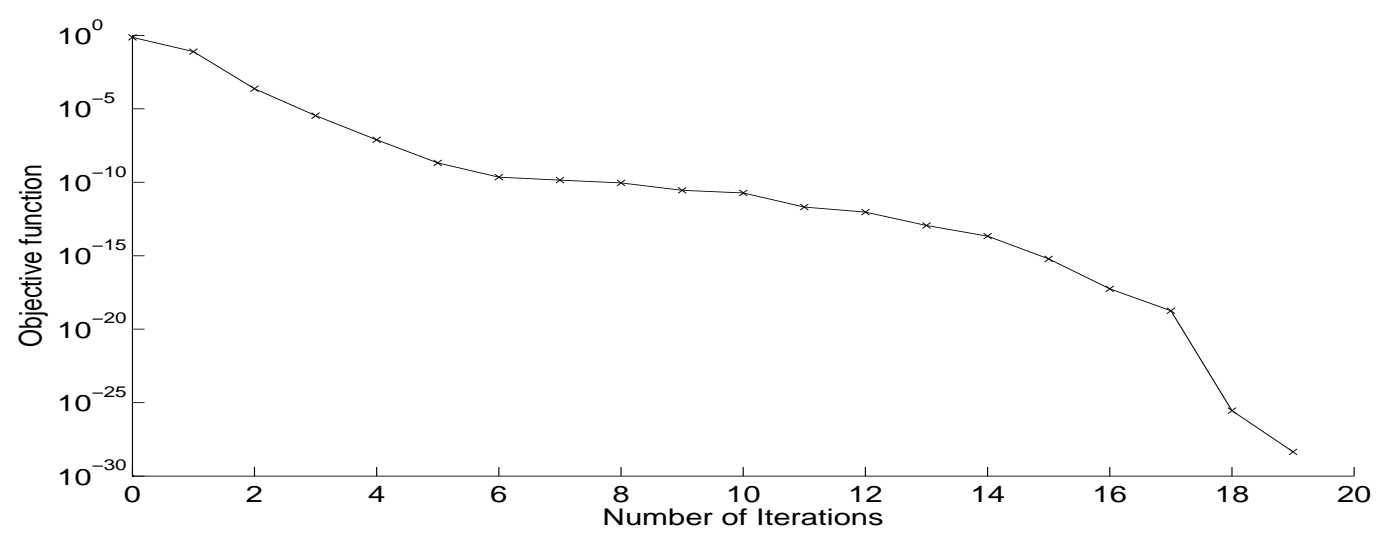

Figure 1: Objective function (43) for Example 1 with no noise and no regularization. 
Next, we add a small amount of $p=0.01 \%$ noise to the measured data (4) and (5). Then we expect that regularization is needed in order to achieve stable and accurate results. The decreasing convergence of the objective function (43), as a function of the number of iterations is shown in Figure 3 with and without regularization. Figure 4 shows the graphs of the recovered functions. From Figure 4 it can be seen that, as expected, when $\beta_{1}=\beta_{2}=0$ we obtain unstable and inaccurate solutions because the problem is ill-posed and very sensitive to noise. Thus regularization is needed in order to stabilise the solutions. We selected by trial and error the regularization parameters $\beta_{1}=10^{-9}$ and $\beta_{2}=10^{-7}$ which give stable and reasonable accurate solutions for the functions $f(t)$ and $g(x)$. For more elaborate choices of multiple regularization parameters, see $[1,5]$.

The related numerical results for the temperature $u(x, t)$ with $p=0.01 \%$ noise, and with and without regularization, are presented in Figure 5 showing good agreement with the exact solution (53).

Other details about number of iterations, the number of function evaluations, the value of the objective function (43) at final iteration, the rmse $(f)$ and $r m s e(g)$ in (48) and (49), respectively, and the computational time are given in Table 1. Overall, from this table as well as from Figures 1-5 it can be seen that accurate and stable numerical results are rapidly achieved by the iterative MATLAB toolbox routine lsqnonlin minimizing the objective function (43).

(a)

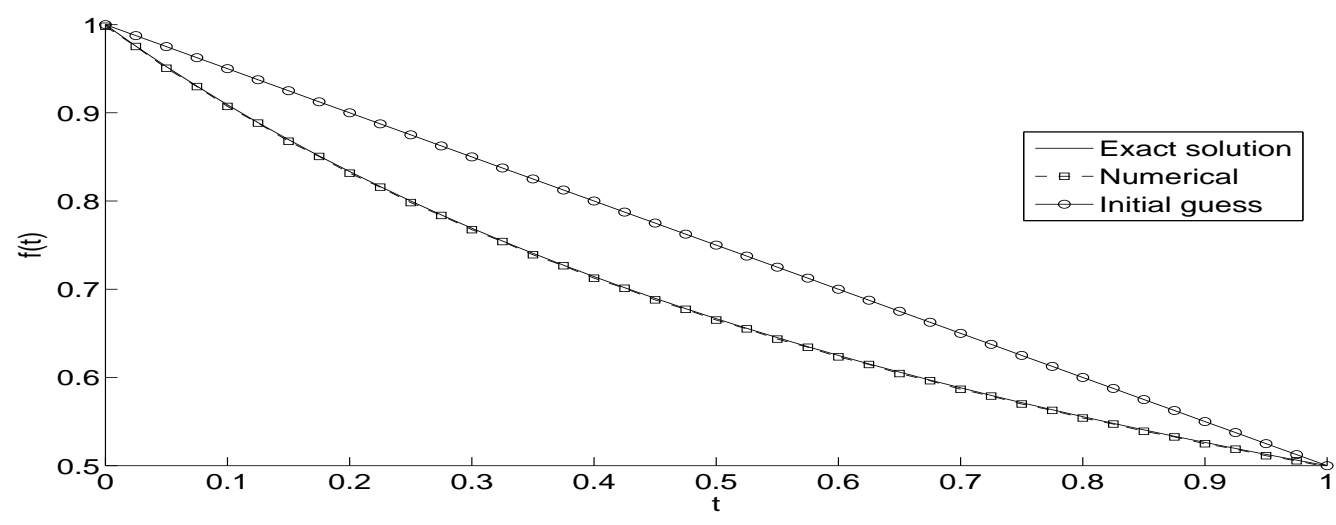

(b)

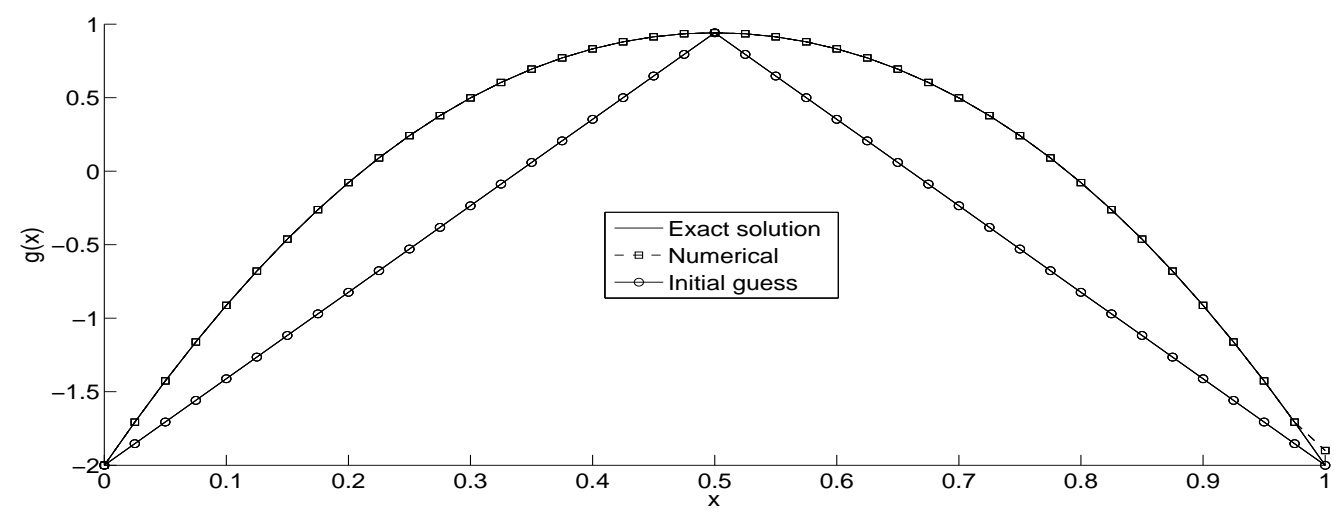

Figure 2: (a) Coefficient $f(t)$ and (b) coefficient $g(x)$, for Example 1 with no noise and no regularization. 
Table 1: Number of iterations, number of function evaluations, value of the objective function (43) at final iteration, $r m s e(f)$ and $r m s e(g)$, and computational time, for Example 1.

\begin{tabular}{|l||c|c|c|}
\hline \multicolumn{1}{|c||}{ Numerical outputs } & $\begin{array}{l}p=0 \\
\left(\beta_{1}=\beta_{2}=0\right)\end{array}$ & $\begin{array}{l}p=0.01 \% \\
\left(\beta_{1}=\beta_{2}=0\right)\end{array}$ & $\begin{array}{c}p=0.01 \% \\
\left(\beta_{1}=10^{-9}, \beta_{2}=10^{-7}\right)\end{array}$ \\
\hline $\begin{array}{l}\text { Number of iterations } \\
\text { Number of function evaluations }\end{array}$ & 19 & 36 & 46 \\
Value of objective function & $4.4 \mathrm{E}-29$ & 3071 & 3901 \\
(43) at final iteration & & $6.5 \mathrm{E}-11$ & $3.2 \mathrm{E}-6$ \\
rmse $(f)$ & 0.0013 & 0.1454 & 0.1024 \\
rmse $(g)$ & 0.0156 & 0.6698 & 0.1634 \\
Computational time (sec) & 163 & 306 & 386 \\
\hline
\end{tabular}

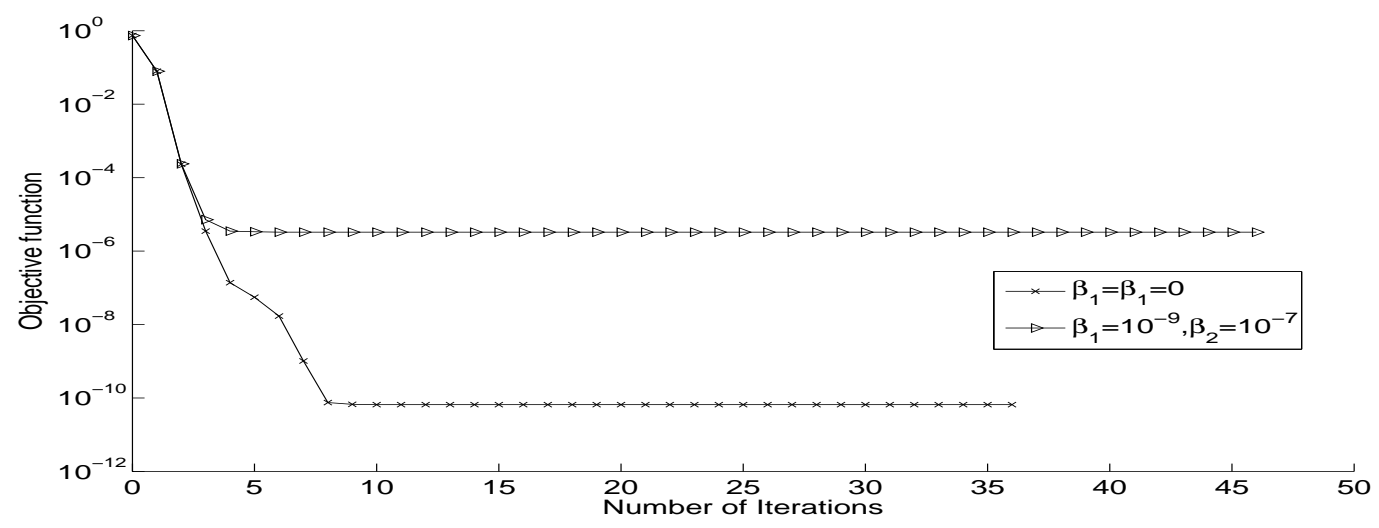

Figure 3: Objective function (43), for Example 1 with $p=0.01 \%$ noise, with and without regularization. 
(a)

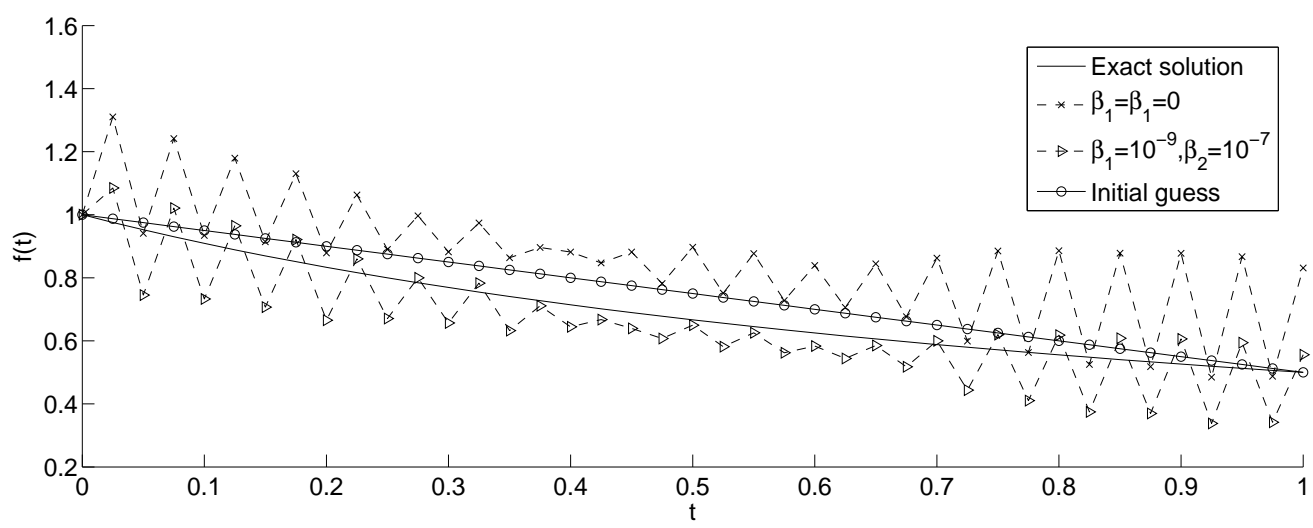

(b)

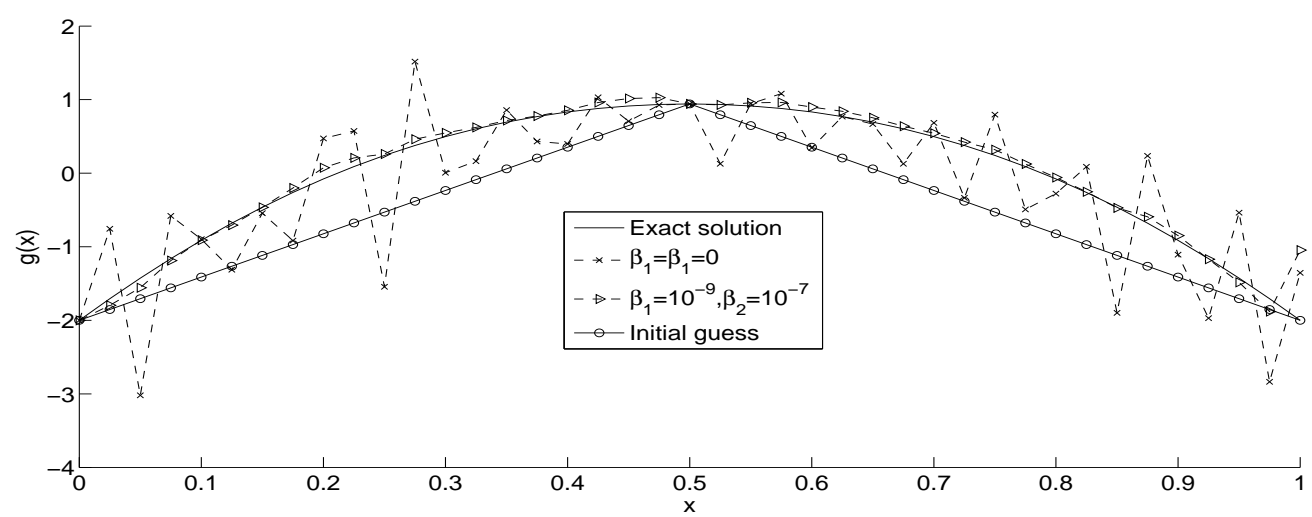

Figure 4: (a) Coefficient $f(t)$ and (b) coefficient $g(x)$, for Example 1 with $p=0.01 \%$ noise, with and without regularization. 
(a)
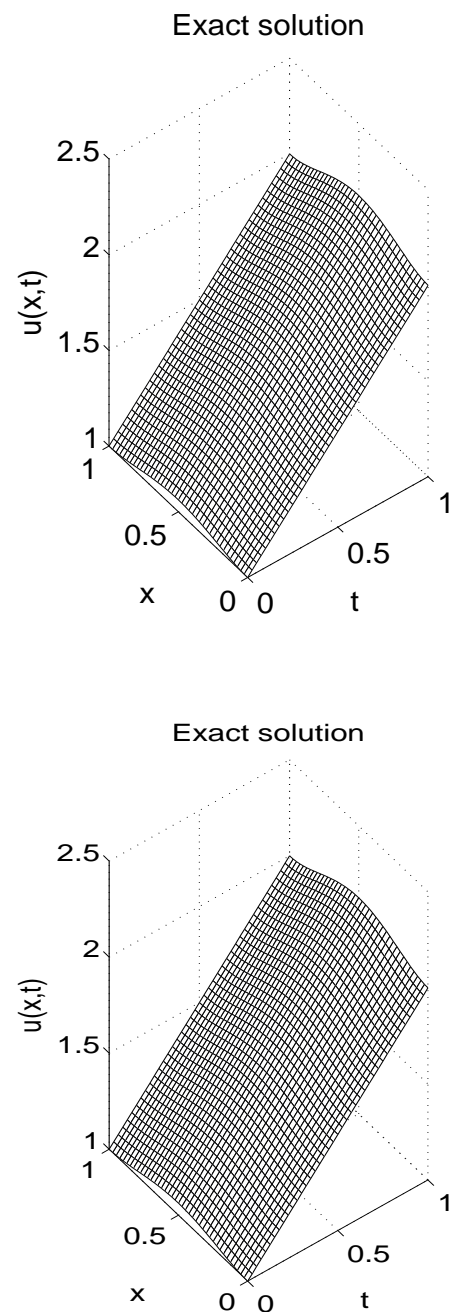

Approximate solution

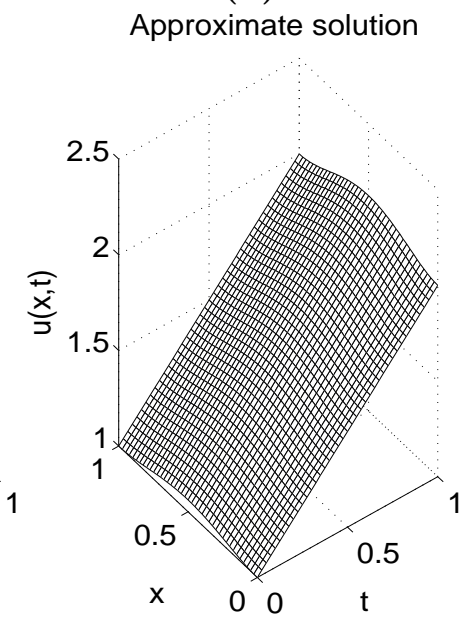

(b)

Approximate solution

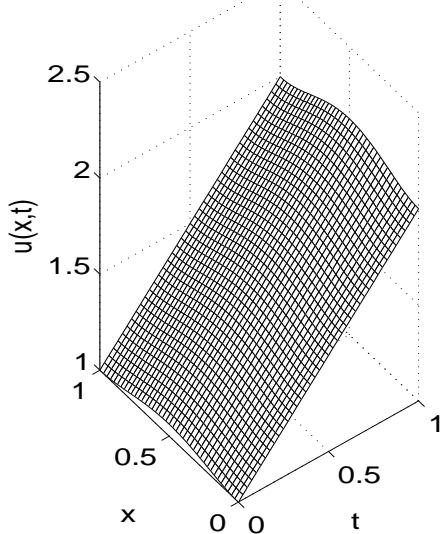

Error graph

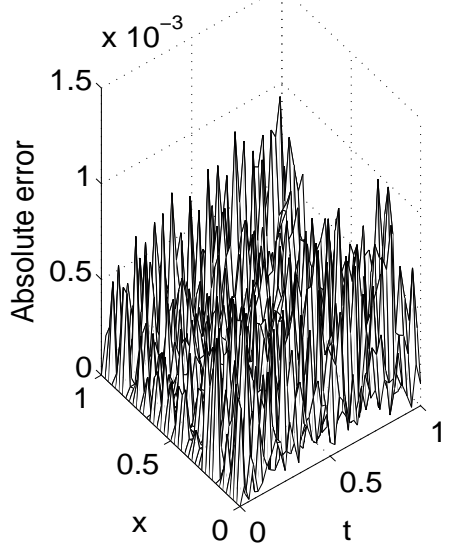

Error graph

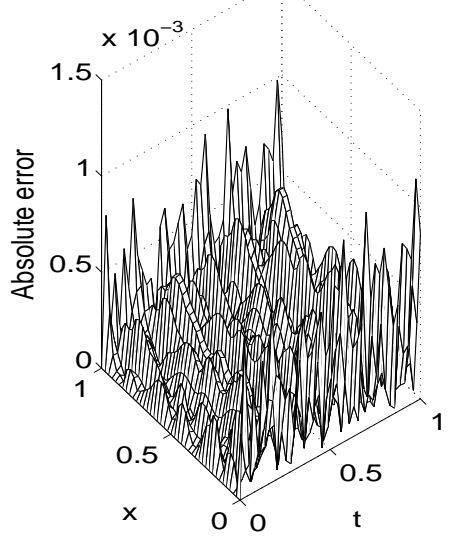

Figure 5: The exact and approximate solutions for the temperatures $u(x, t)$, for Example 1, with $(a) \beta_{1}=\beta_{2}=0$ and $(b) \beta_{1}=10^{-9}, \beta_{2}=10^{-7}$, for $p=0.01 \%$ noise. The absolute error between them is also included.

\subsection{Example 2}

Consider the inverse problem (1)-(7) with the input data

$$
\begin{gathered}
\phi(x)=u(x, 0)=2+\cos (\pi x), \\
\beta(t)=u(0.5, t)=2 e^{\frac{t^{2}}{1+t}}, \quad \psi(x)=u(x, 1)=\sqrt{e}(2+\cos (\pi x)), \quad \alpha=g(0.5)=0 .
\end{gathered}
$$

As in Example 1, the conditions of Proposition 1 are satisfied and the unique solution of the inverse problem (1)-(7) is given by (10)-(12) which yield

$$
\begin{gathered}
f(t)=\frac{t(t+2)}{(t+1)^{2}}, \quad g(x)=\frac{\pi^{2} \cos (\pi x)}{2+\cos (\pi x)}, \\
u(x, t)=e^{\frac{t^{2}}{1+t}}(2+\cos (\pi x)) .
\end{gathered}
$$


We take the inital guess as

$$
f^{0}(t)=0, \quad g^{0}(x)= \begin{cases}\frac{\pi^{2}}{3}-\frac{2 \pi^{2}}{3} x, & 0 \leq x \leq 0.5 \\ \pi^{2}-2 \pi^{2} x, & 0.5<x \leq 1\end{cases}
$$

Analogous quantities and conclusions to Figures 1-4 and Table 1 of Example 1 are presented and obtained in Figures 6-9 and Table 2 of Example 2.

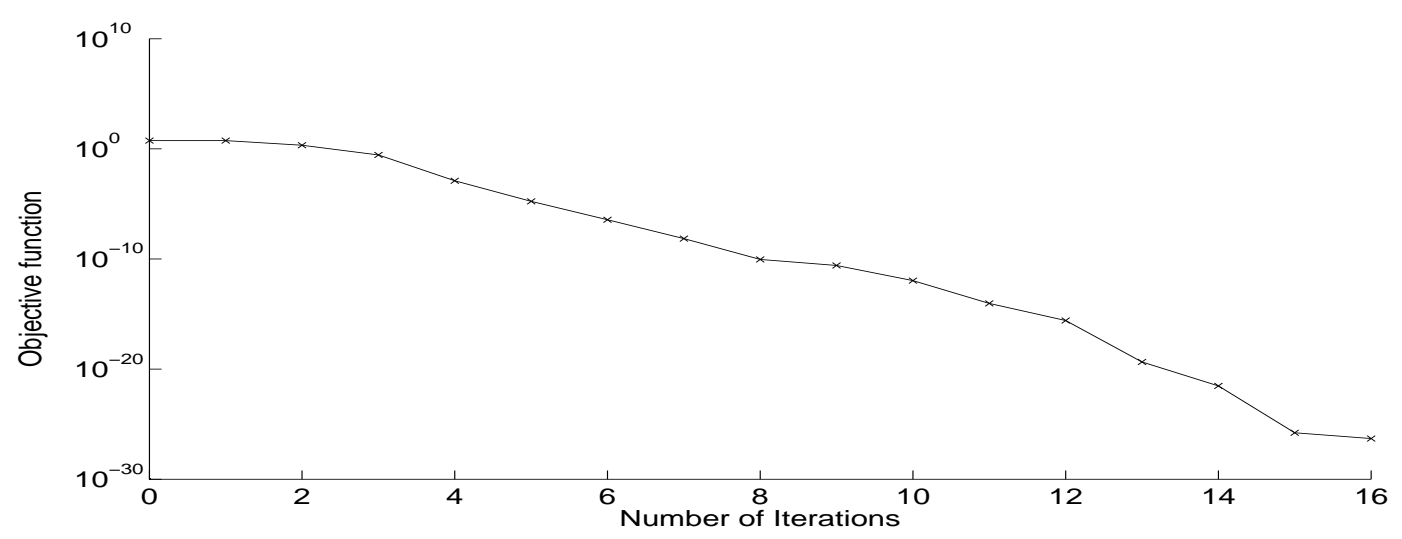

Figure 6: Objective function (43), for Example 2 with no noise and no regularization.

(a)

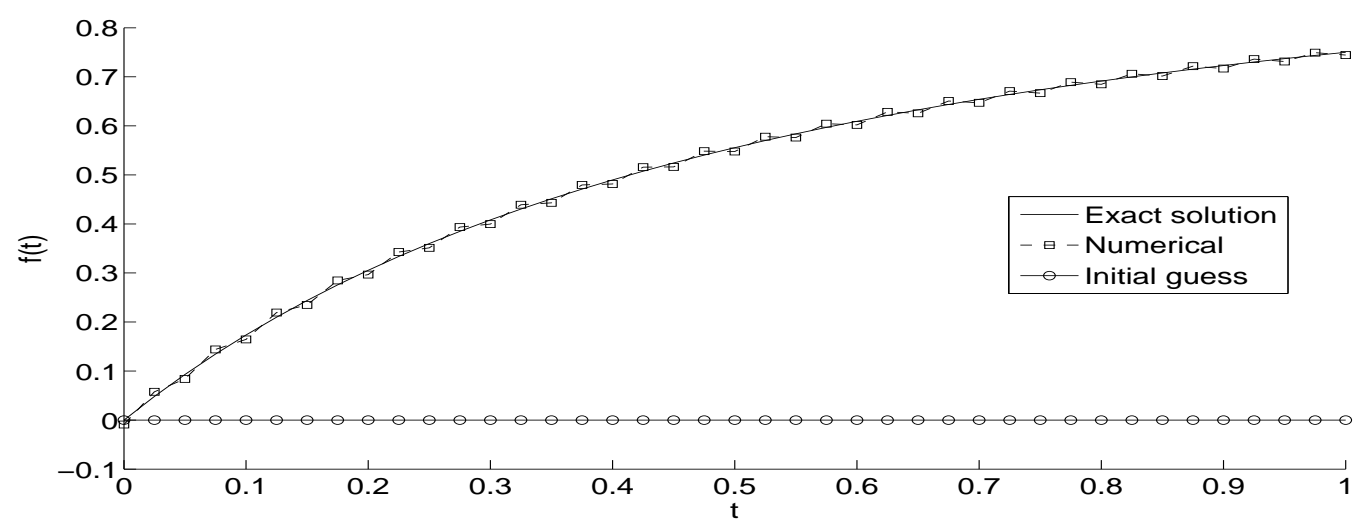

(b)

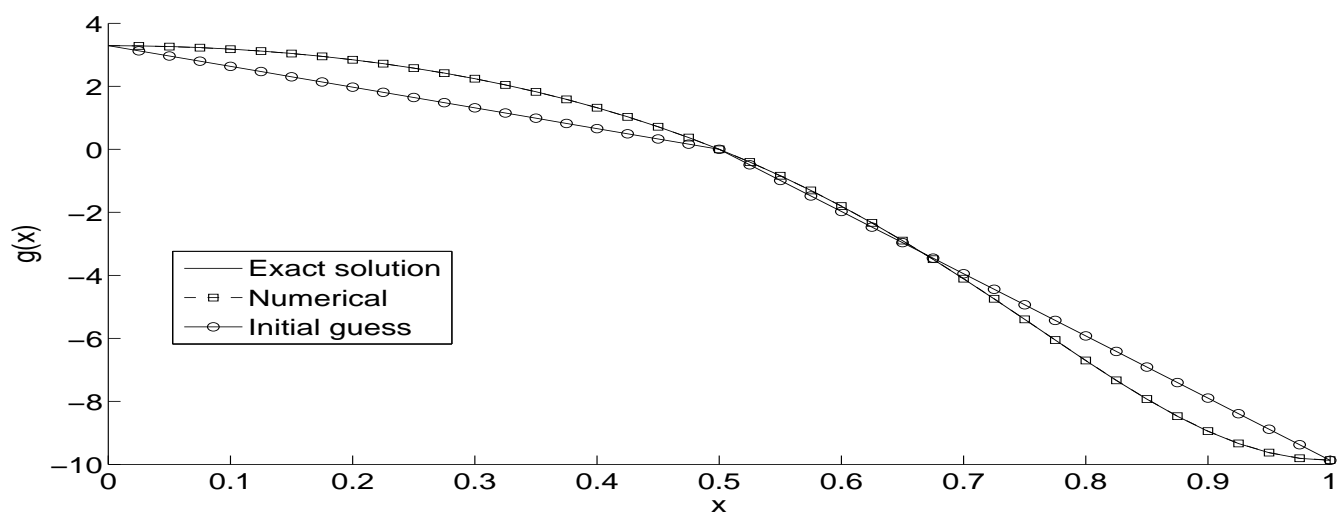

Figure 7: (a) Coefficient $f(t)$ and (b) coefficient $g(x)$, for Example 2 with no noise and no regularization. 
Table 2: Number of iterations, number of function evaluations, value of the objective function (43) at final iteration, $r m s e(f)$ and $r m s e(g)$, and computational time, for Example 2 .

\begin{tabular}{|l||c|c|c|}
\hline \multicolumn{1}{|c|}{ Numerical outputs } & $\begin{array}{l}p=0 \\
\left(\beta_{1}=\beta_{2}=0\right)\end{array}$ & $\begin{array}{l}p=0.01 \% \\
\left(\beta_{1}=\beta_{2}=0\right)\end{array}$ & $\begin{array}{c}p=0.01 \% \\
\left(\beta_{1}=10^{-12}, \beta_{2}=10^{-11}\right)\end{array}$ \\
\hline Number of iterations & 16 & 39 & 52 \\
Number of function evaluations & 1411 & 3320 & 4316 \\
Value of objective function & $4.9 \mathrm{E}-27$ & $1.5 \mathrm{E}-10$ & $1.02 \mathrm{E}-8$ \\
(43) at final iteration & & & \\
rmse $(f)$ & 0.0077 & 0.1462 & 0.1084 \\
rmse $(g)$ & 0.0025 & 1.2917 & 1.2847 \\
Computational time (mins) & 2 & 5 & 7 \\
\hline
\end{tabular}

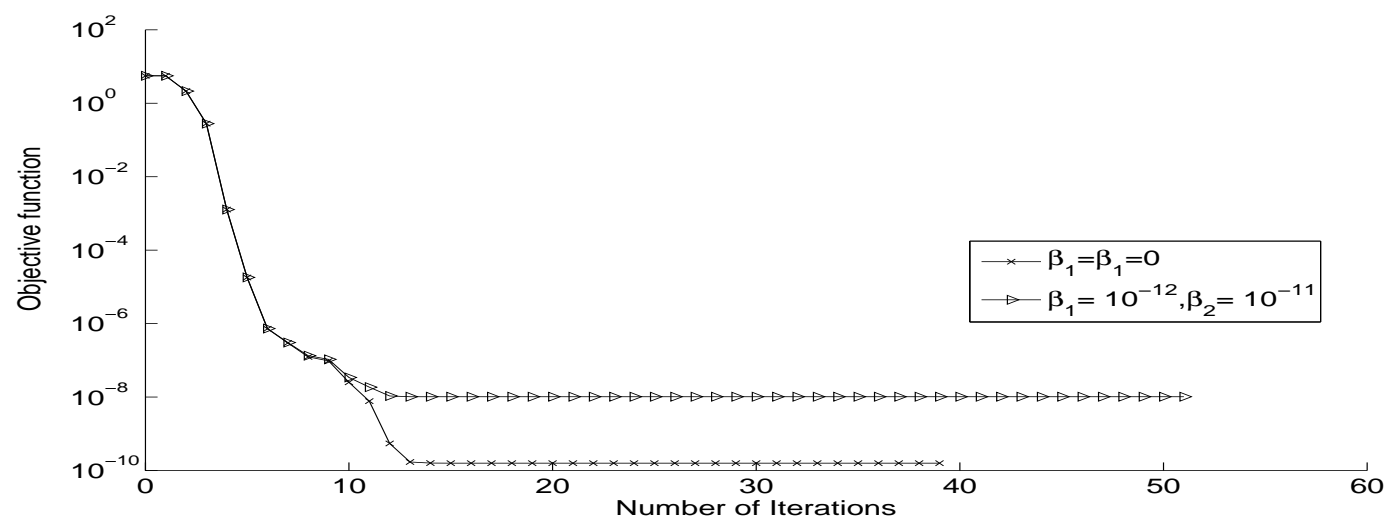

Figure 8: Objective function (43), for Example 2 with $p=0.01 \%$ noise, with and without regularization. 
(a)

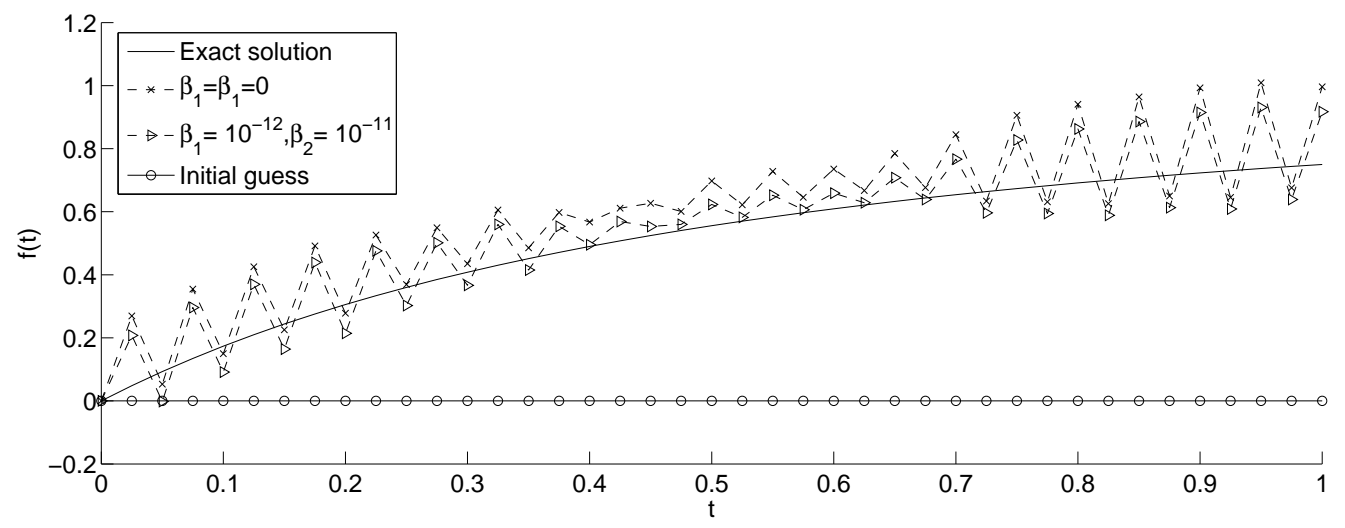

(b)

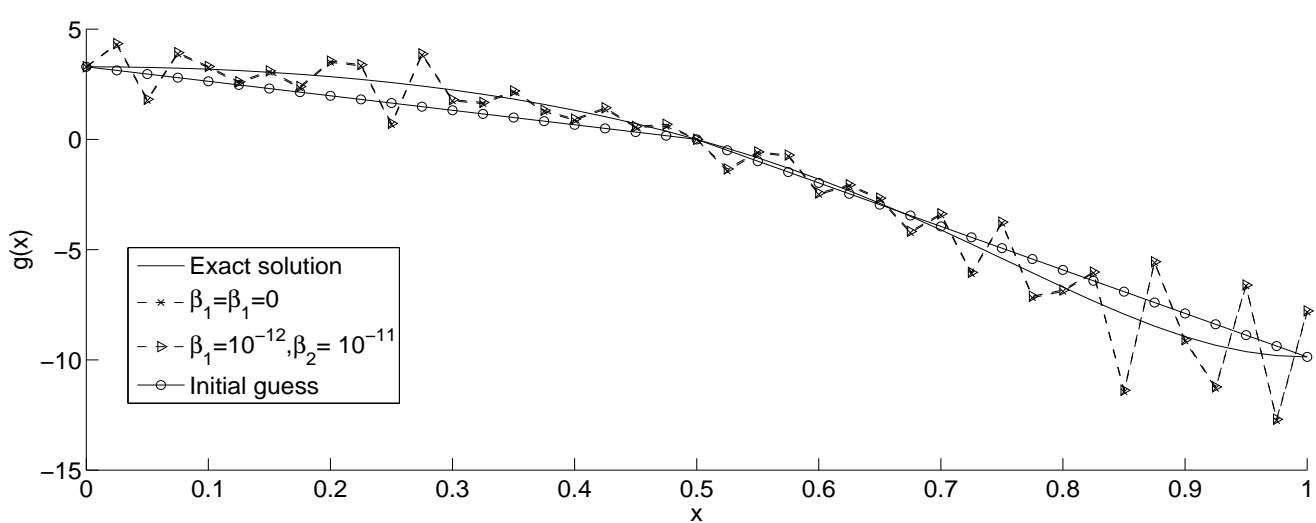

Figure 9: (a) Coefficient $f(t)$ and (b) coefficient $g(x)$, for Example 2 with $p=0.01 \%$ noise, with and without regularization.

\subsection{Example 3}

The previous examples possessed an analytical solution available for the triple $(u(x, t), f(t), g(x))$. In this section, we investigate an example for which an analytical solution for $u(x, t)$ is not available. We take the initial condition (2) given by

$$
u(x, 0)=\phi(x)=\left\{\begin{array}{lr}
1, & 0 \leq x<1 / 4 \\
\frac{5}{4}-x, & 1 / 4<x \leq 1 / 2, \\
x+\frac{1}{4}, & 1 / 2<x \leq 3 / 4 \\
1, & 3 / 4<x \leq 1
\end{array}\right.
$$

which represents a non-smooth function. In the absence of an analytical solution for $u(x, t)$ being available we generate the input data (4) and (5) numerically by solving first the direct problem given by (1)-(3) with $\phi(x)$ given by (60), and the known functions

$$
f(t)=1+t, \quad g(x)=1+x,
$$

using the FDM described in Section 3.

The numerical results for $\beta(t)$ and $\psi(x)$ in equations (4) and (5), respectivly, are shown in Figure 10, for various $M=N \in\{20,40,80\}$. From this figure, it can be seen that the numerical solution is convergent as the FDM mesh size decreases. 
In order to avoid committing an inverse crime the mesh that is used for numerically simulating the measured data (4) and (5) by solving the direct problem is more dense than the one used for the solution of the inverse problem, [8]. Consequently, in the inverse problem we use the FDM with $M=N=40$ and half of the data for $\beta$ and $\psi$ obtained from solving the direct problem with $M=N=80$. We also take the initial guess as

$$
f^{0}(t)=1, \quad g^{0}(x)=1 .
$$

The objective function (43) with no noise and no regularization, as a function of the number of iterations, is plotted in Figure 11. From this figure it can be seen that a rapid monotonic decrease to a low value of $O\left(10^{-28}\right)$ is achieved in about 12 iterations.

(a)

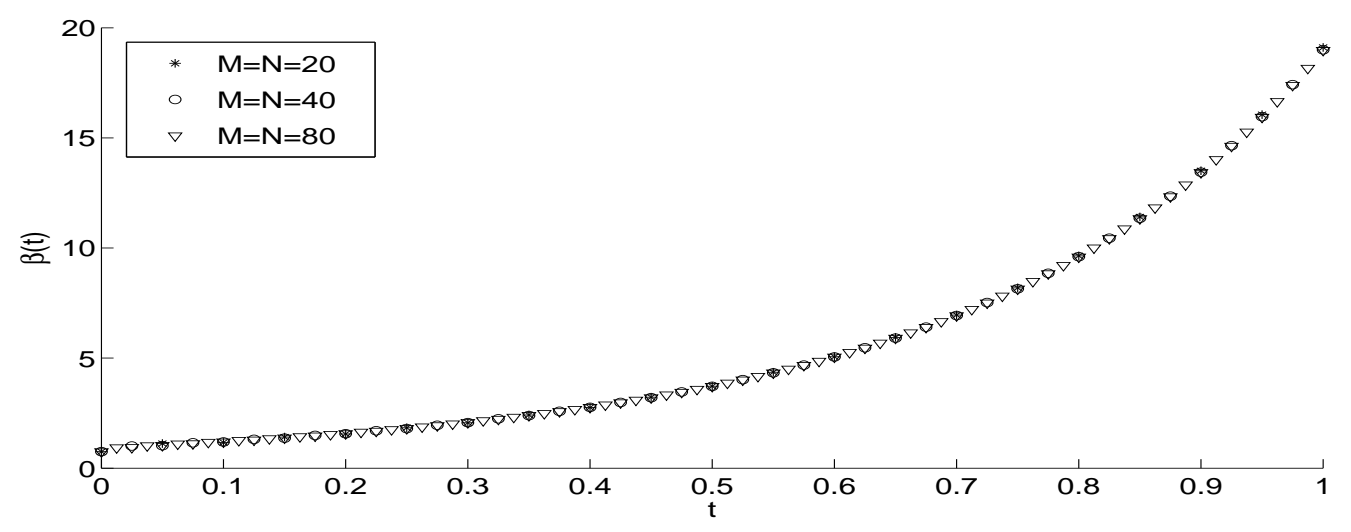

(b)

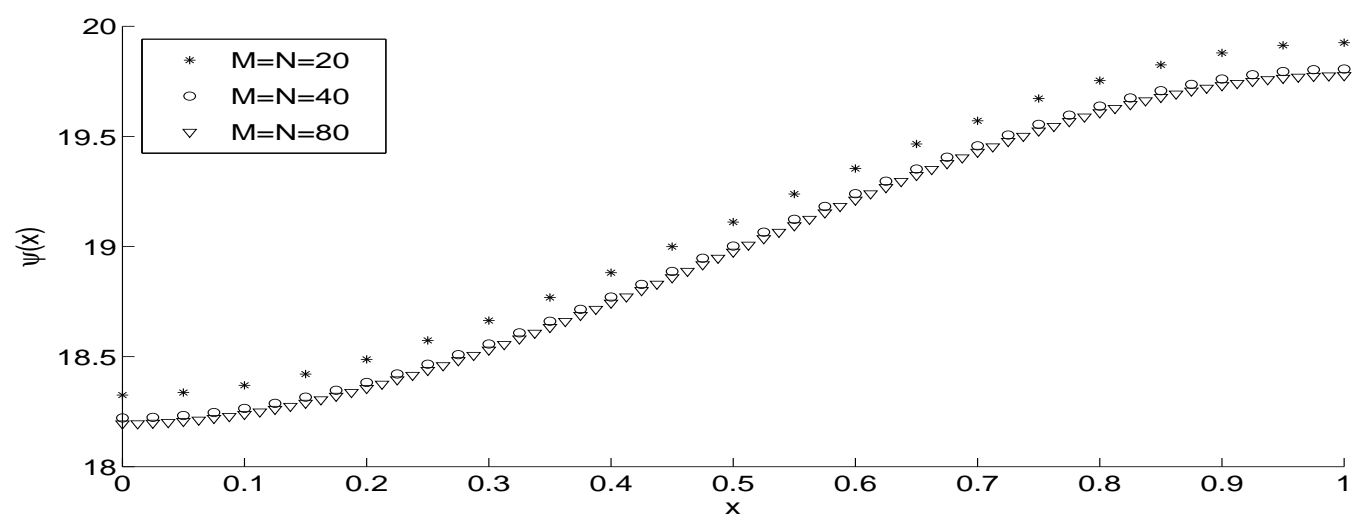

Figure 10: (a) The numerically convergent solutions for $\beta(t)$ and (b) $\psi(x)$, for Example 3 with various mesh sizes $M=N \in\{20,40,80\}$ for the direct problem.

Figure 12 shows the exact and numerical solution of the unknown coefficients $f(t)$ and $g(x)$, and one can see that the stable and accurate solutions are obtained. 


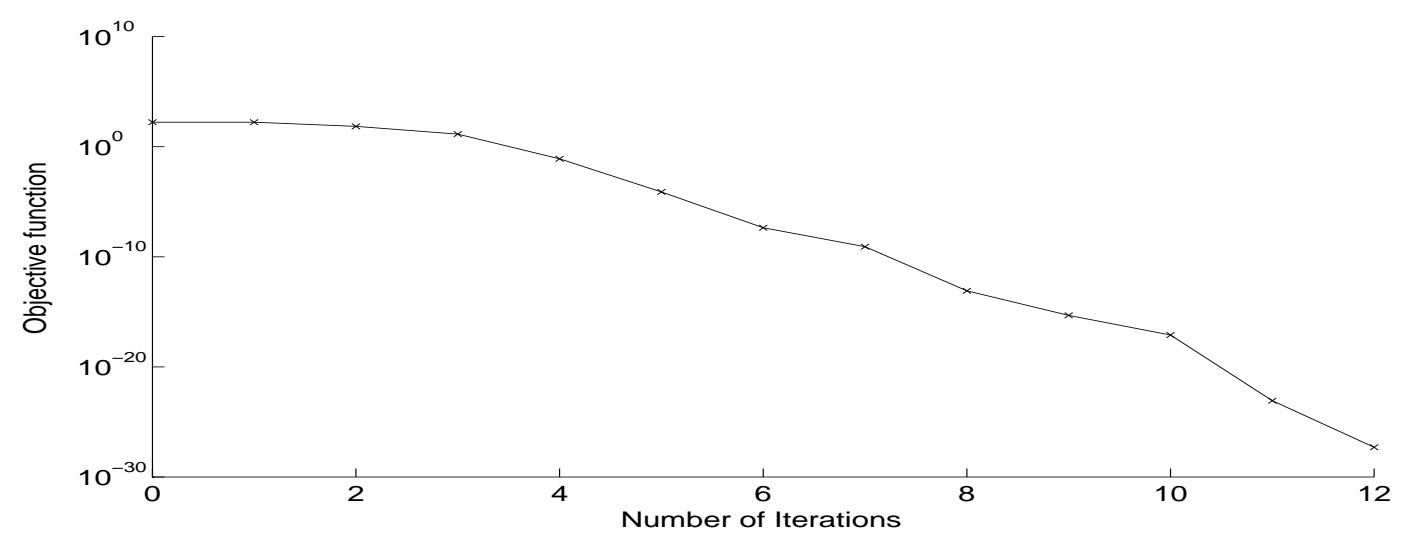

Figure 11: Objective function (43), for Example 3 with no noise and no regularization.

(a)

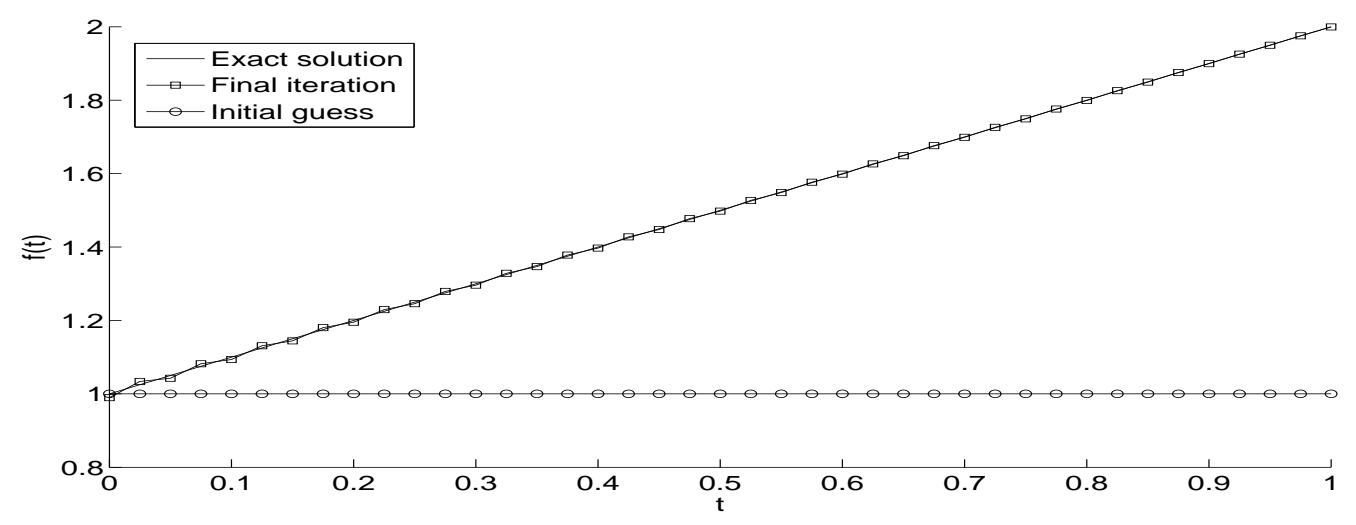

(b)

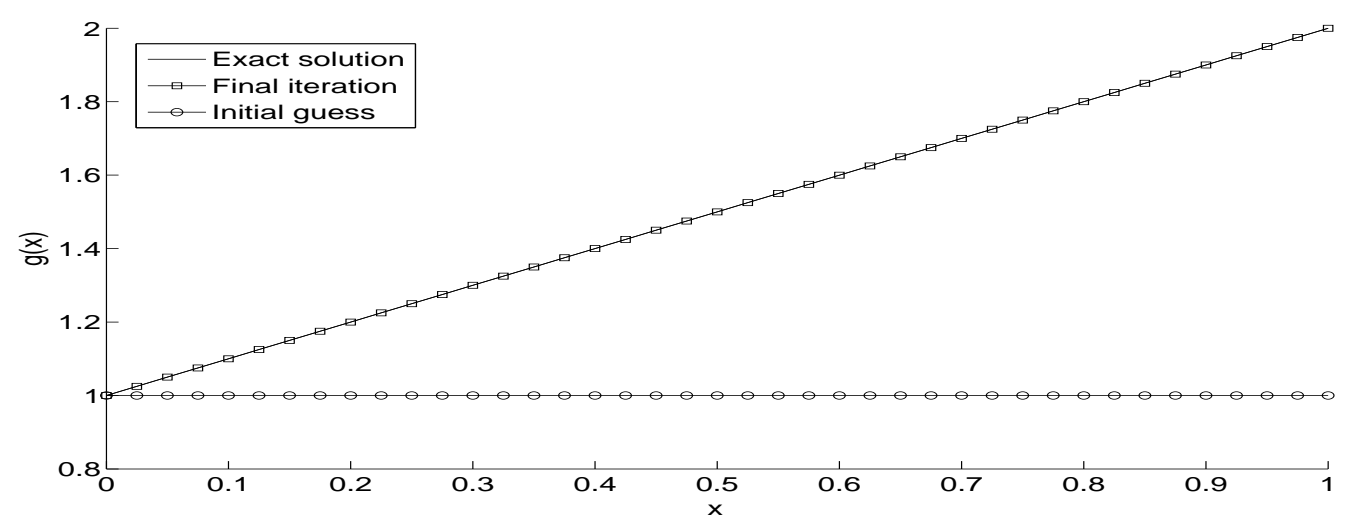

Figure 12: (a) Coefficient $f(t)$ and (b) coefficient $g(x)$, for Example 3 with no noise and no regularization.

Next, we add $p=0.01 \%$ noise to the measurements data (4) and (5). The objective function (43), as a function of the number of iterations is shown in Figure 13 with and without regularization. Figure 14 presents the graphs of the recovered functions. From this figure one can observe that, as expected, when $\beta_{1}=\beta_{2}=0$ we obtain unstable and inaccurate solutions because the problem is ill-posed and very sensitive to noise. So, regularization is needed in order to stabilize the solution which is achieved by selecting $\beta_{1}=\beta_{2}=10^{-5}$, as illustrated in Figure 14 . 
Table 3: Number of iterations, number of function evaluations, value of the objective function (43) at final iteration, $r m s e(f)$ and $r m s e(g)$, and computational time, for Example 3 .

\begin{tabular}{|l||c|c|c|}
\hline \multicolumn{1}{|c||}{ Numerical outputs } & $\begin{array}{l}p=0 \\
\left(\beta_{1}=\beta_{2}=0\right)\end{array}$ & $\begin{array}{l}p=0.01 \% \\
\left(\beta_{1}=\beta_{2}=0\right)\end{array}$ & $\begin{array}{c}p=0.01 \% \\
\left(\beta_{1}=\beta_{2}=10^{-5}\right)\end{array}$ \\
\hline $\begin{array}{l}\text { Number of iterations } \\
\text { Number of function evaluations }\end{array}$ & 12 & 18 & 42 \\
Value of objective function & $5.00 \mathrm{E}-28$ & $5.2 \mathrm{E}-9$ & 3486 \\
(43) at final iteration & & & $1.9 \mathrm{E}-3$ \\
rmse $(f)$ & 0.0036 & 0.3373 & 0.0446 \\
rmse $(g)$ & $1.4 \mathrm{E}-6$ & 0.5775 & 0.1424 \\
Computational time (sec) & 95 & 141 & 343 \\
\hline
\end{tabular}

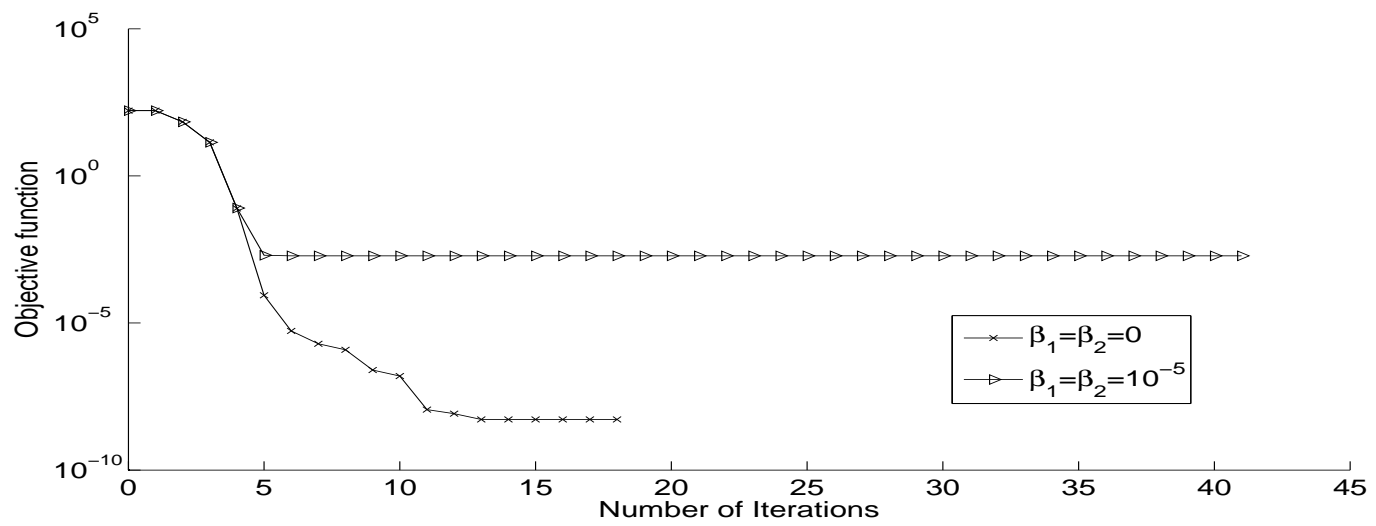

Figure 13: Objective function (43), for Example 3 with $p=0.01 \%$ noise, with and without regularization. 
(a)

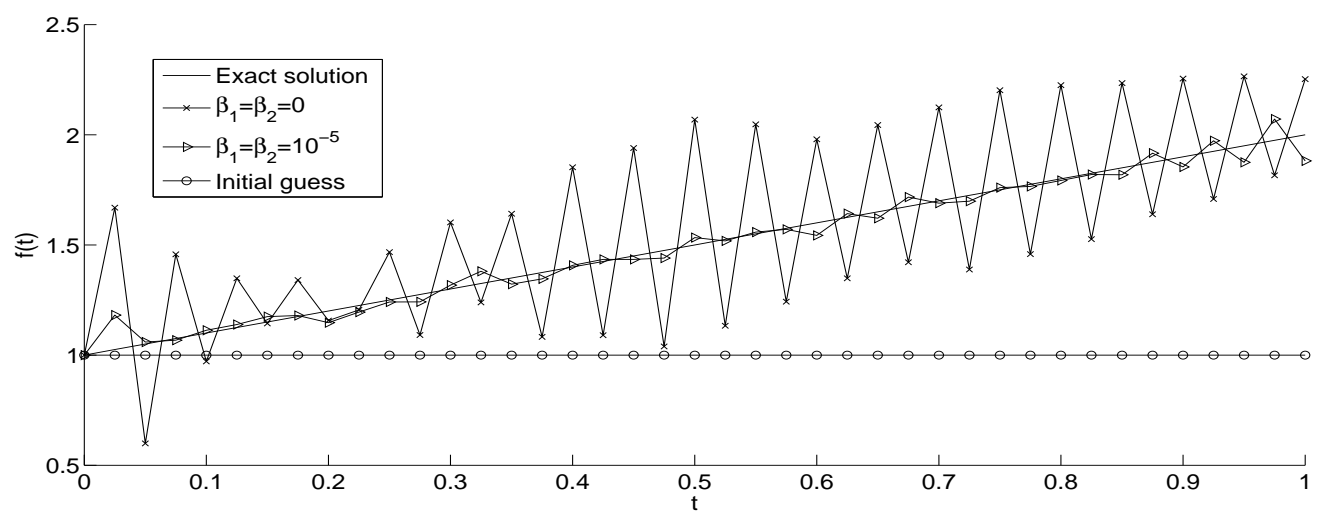

(b)

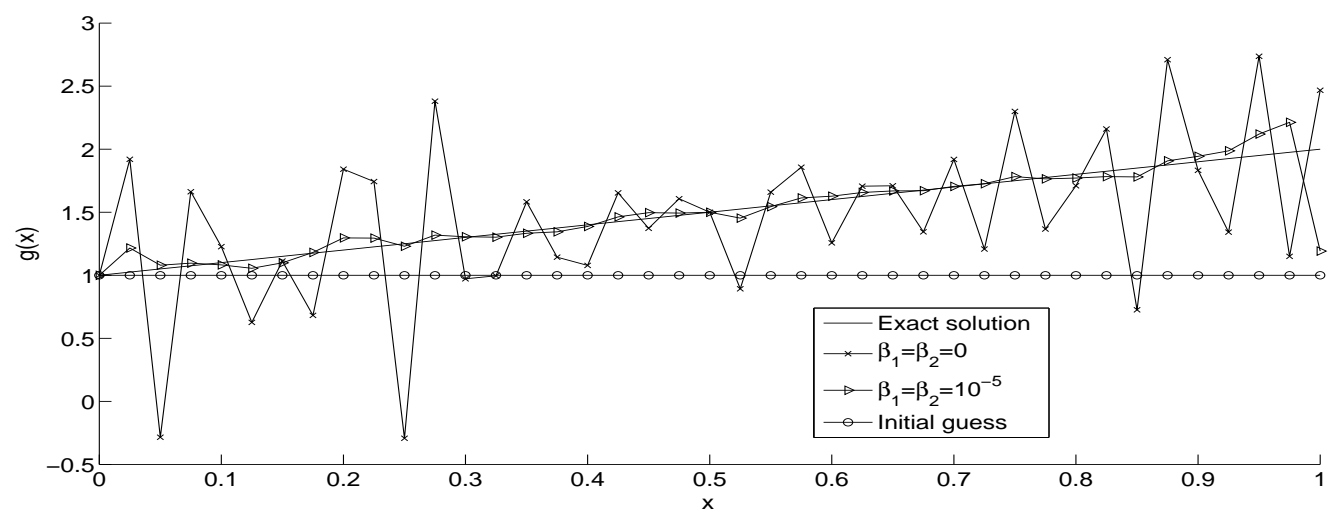

Figure 14: (a) Coefficient $f(t)$ and (b) coefficient $g(x)$, for Example 3 with $p=0.01 \%$ noise, with and without regularization.

Finally, the other details about number of iterations, the number of function evaluations, the value of the objective function (43) at final iteration, the rmse $(f)$ and $r m s e(g)$ in (48) and (49), respectively, and the computational time, are given in Table 3. From this table it can be seen that accurate and stable numerical results are achieved if regularization is employed when inverting noisy data.

\section{Conclusions}

This paper has presented the determination of the time and space-dependent perfusion coefficient from data measurements in the one-dimensional parabolic heat equation. The direct solver based on a Crank-Nicolson finite difference scheme was employed. The resulting inverse problem has been reformulated as a constrained regularized minimization problem which was solved using the MATLAB optimization toolbox routine lsqnonlin. The numerically obtained results are shown to be stable and accurate.

\section{Acknowledgments}

M.J. Huntul would like to thank Jazan University in Saudi Arabia and United Kingdom Saudi Arabian Cultural Bureau (UKSACB) in London for financing his PhD at the 
University of Leeds.

\section{References}

[1] Belge, M., Kilmer, M. and Miller, E. (2002) Efficient determination of multiple regularization parameters in a generalized L-curve framework, Inverse Problems, 18, $1161-1183$.

[2] Coleman, T.F. and Li, Y. (1996) An interior trust region approach for nonlinear minimization subject to bounds, SIAM Journal on Optimization, 6, 418-445.

[3] Deng, Z.C., Yang, L., Yu, J.N. and Luo, G.W. (2010) Identifying the radiative coefficient of an evolutional type heat conduction equation by optimization method, Journal of Mathematical Analysis and Applications, 362, 210-223.

[4] Deng, Z.C., Yu, J.N. and Yang, L. (2008) Optimization method for an evolutional type inverse heat conduction problem, Journal of Physics A: Mathematical and Theoretical, 41, 035201, (20 pp).

[5] Fornasier, M., Naumova, V. and Pereverzyev, S.V. (2014) Parameter choice strategies for multipenalty regularization, SIAM Journal on Numerical Analysis, 52, 17701794.

[6] Hazanee, A. and Lesnic, D. (2013) Reconstruction of an additive space- and timedependent heat source, European Journal of Computational Mechanics, 22, 304-329.

[7] Hao Dinh Nho, Thanh Phan Xuan, Lesnic, D. and Ivanchov, M. (2014) Determination of a source in the heat equation from integral observations, Journal of Computational and Applied Mathematics, 264, 82-98.

[8] Kaipio, J. and Somersalo, E. (2007) Statistical inverse problems: discretization, model reduction and inverse crimes, Journal of Computational and Applied Mathematics, 198, 493-504.

[9] Mathwoks R2012 Documentation Optimization Toolbox-Least Squares (Model Fitting) Algorithms, available from www.mathworks.com/help/toolbox/optim/ug /brnoybu.html.

[10] Samarskii, A.A. and Vabishchevich, P.N. (2007) Numerical Methods for Solving Inverse Problems of Mathematical Physics, De Gruyter, Berlin.

[11] Savateev, E.G. (1995) On the problem of identification of a coefficient in a parabolic equation. Siberian Mathematical Journal, 36, 177-185.

[12] Smith, G.D. (1985) Numerical Solution of Partial Differential Equations: Finite Difference Methods, Oxford Applied Mathematics and Computing Science Series, Third edition.

[13] Trucu, D. (2009) Inverse Problems for Blood Perfusion Identification, PhD Thesis, University of Leeds. 
[14] Trucu, D., Ingham, D.B. and Lesnic, D. (2011) Reconstruction of the space- and time-dependent blood perfusion coefficient in bio-heat transfer, Heat Transfer Engineering, 32, 800-810.

\section{Appendix A}

Take $X_{0}=\pi$ and throughout we shall use Wirtinger's inequality

$$
\|h\|_{L^{2}(0, \pi)} \leq\left\|h^{\prime}\right\|_{L^{2}(0, \pi)}, \quad \forall h \in C^{1}[0, \pi] \text { with } h(0)=h(\pi)=0 .
$$

Inverse Problem: Let $T>0$ and denote $Q_{T}=(0, \pi) \times(0, T)$. Find the triplet

$$
(u(x, t), f(t), g(x)) \in\left(C^{2}\left(Q_{T}\right) \cap C^{1}\left(\overline{Q_{T}}\right)\right) \times C^{1}[0, T] \times C^{1}[0, \pi]=: \mathcal{S}
$$

satisfying the problem

$$
\begin{gathered}
u_{t}=u_{x x}+(f(t)+g(x)) u, \quad(x, t) \in Q_{T}, \\
u(x, 0)=\phi(x), \quad x \in[0, \pi], \\
u_{x}(0, t)=u_{x}(\pi, t)=0, \quad t \in[0, T], \\
u(x, T)=\psi(x), \quad x \in[0, \pi], \\
u\left(X_{0}, t\right)=\beta(t), \quad t \in[0, T], \\
g\left(X_{1}\right)=\alpha,
\end{gathered}
$$

where $X_{0}$ and $X_{1}$ are fixed points in $(0, \pi)$.

We suppose that the input data (A.4)-(A.7) satisfy the compatibility conditions

$$
\phi^{\prime}(0)=\phi^{\prime}(\pi)=\psi^{\prime}(0)=\psi^{\prime}(\pi)=0, \quad \beta(0)=\phi\left(X_{0}\right), \quad \beta(T)=\psi\left(X_{0}\right) .
$$

Theorem 1. (Uniqueness)

Suppose $\phi$ and $\psi \in C^{4}[0, \pi]$ and $\beta \in C^{1}[0, T]$ satisfy $\phi>0, \psi>0$ and $\beta>0$ and the compatibility conditions (A.9). Assume further that

$$
\text { ??? }
$$

Then the inverse problem (A.3)-(A.8) has at most one classical solution in the space $\mathcal{S}$ defined in (A.2). 
Lemma 1. Let $z_{1} \geq 0, a \in(0,1), c \geq 0, b>0$ satisfy

$$
\begin{gathered}
4 b c \leq(1-a)^{2}, \\
\frac{1-a-\sqrt{(1-a)^{2}-4 b c}}{2 b} \leq z_{1} \leq \frac{1-a+\sqrt{(1-a)^{2}-4 b c}}{2 b} .
\end{gathered}
$$

Define the sequence

$$
z_{n+1}=a z_{n}+b z_{n}^{2}+c, \quad n \geq 1
$$

Then $\left(z_{n}\right)_{n \geq 1}$ is a decreasing sequence and, in particular,

$$
0 \leq z_{n} \leq z_{1}, \quad n \geq 1 \text {. }
$$

Proof: Let $n=1$ in (A.13) to obtain

$$
z_{2}=a z_{1}+b z_{1}^{2}+c .
$$

Using (A.13) and (A.12) it follows that $0 \leq z_{2} \leq z_{1}$. Let $n \geq 2$ and suppose by mathemaical induction that $z_{n} \leq z_{n-1}$ and wish to show that $z_{n+1} \leq z_{n}$.

From (A.13) and $z_{n} \leq z_{n-1}$ we have that

$$
z_{n+1}=a z_{n}+b z_{n}^{2}+c \leq a z_{n-1}+b z_{n-1}^{2}+c=z_{n},
$$

which concludes the proof of lemma.

\section{Proof of Theorem 1.}

We follow Savateev (1995) and first remark that from the maximum principle, the Harnack inequality and hypotheses of the theorem we can demonstrate that any smooth solution to the inverse problem satisfies

$$
u>0 \text { in } \overline{Q_{T}} .
$$

Then we can define

$$
u=e^{v}
$$

and, for the new function $v$, equations (A.3)-(A.7) recast as

$$
\begin{gathered}
v_{t}=v_{x x}+v_{x}^{2}+f(t)+g(x), \quad(x, t) \in Q_{T}, \\
v(x, 0)=\Phi(x):=\ln (\phi(x)), \quad x \in[0, \pi], \\
v_{x}(0, t)=v_{x}(\pi, t)=0, \quad t \in[0, T], \\
v(x, T)=\Psi(x):=\ln (\psi(x)), \quad x \in[0, \pi], \\
v\left(X_{0}, t\right)=\beta_{1}(t):=\ln (\beta(t)), \quad t \in[0, T] .
\end{gathered}
$$


Differentiating (A.17) with respect to $x$ we eliminate $f(t)$ and obtain

$$
v_{t x}=v_{x x x}+2 v_{x} v_{x x}+g^{\prime}(x), \quad(x, t) \in Q_{T} .
$$

Differentiating (A.22) with respect to $t$ we eliminate $g^{\prime}(x)$ and obtain

$$
\begin{array}{r}
w_{t}=w_{x x}+2\left(\int_{T}^{t} w(x, \tau) d \tau+\Psi^{\prime}(x)\right) w_{x}+2\left(\int_{T}^{t} w_{x}(x, \tau) d \tau+\Psi^{\prime \prime}(x)\right) w \\
(x, t) \in Q_{T}
\end{array}
$$

where

$$
w=v_{t x} .
$$

Gathering (A.18)-(A.20) and (A.22) we can obtain:

$$
\begin{gathered}
w(x, T)-w(x, 0)=\theta(x), \quad x \in[0, \pi], \\
w(0, t)=w(\pi, t)=0, \quad t \in[0, T],
\end{gathered}
$$

where

$$
\theta(x)=\frac{d}{d x}\left[\frac{\psi^{\prime \prime}(x)}{\psi(x)}-\frac{\phi^{\prime \prime}(x)}{\phi(x)}\right]
$$

With the unknown functions $f(t)$ and $g(x)$ eliminated we shall now show that the problem (A.23), (A.25) and (A.26) has a unique solution.

Set $N:=w^{(1)}-w^{(2)}$, where $w^{(i)}, i=1,2$ are two solutions of (A.23), (A.25) and (A.26) and let us show that $N \equiv 0$. We have that $N$ satisfies the problem

$$
\begin{gathered}
N_{t}=N_{x x}+2\left(w_{x}^{(1)} \int_{T}^{t} N(x, \tau) d \tau+N_{x} \int_{T}^{t} w^{(2)}(x, \tau) d \tau\right. \\
\left.+w^{(1)} \int_{T}^{t} N_{x}(x, \tau) d \tau+N \int_{T}^{t} w_{x}^{(2)}(x, \tau) d \tau\right), \quad(x, t) \in Q_{T}, \\
N(x, T)-N(x, 0)=0, \quad x \in[0, \pi], \\
N(0, t)=N(\pi, t)=0, \quad t \in[0, T] .
\end{gathered}
$$

Multiply (A.28) with $N$ and integrate over $Q_{T}$ to obtain, using (A.26), (A.29) and (A.30), that

$$
\begin{array}{r}
\int_{Q_{T}} N_{x}^{2}(x, t) d x d t=2 \int_{0}^{T} \int_{0}^{\pi} N_{x}(x, t)\left[w^{(1)}(x, t) \int_{t}^{T} N(x, \tau) d \tau\right. \\
\left.+N(x, t) \int_{t}^{T} w^{(2)}(x, \tau) d \tau\right] d x d t
\end{array}
$$

where we have used that (A.28) can be rewritten as

$$
N_{t}=N_{x x}+2\left[\frac{\partial}{\partial x}\left(w^{(1)}(x, t) \int_{T}^{t} N(x, \tau) d \tau\right)+\frac{\partial}{\partial x}\left(N(x, t) \int_{T}^{t} w^{(2)}(x, \tau) d \tau\right)\right]
$$


and integration by parts. Let us first rewrite (A.31) as

$$
\frac{1}{2} \int_{Q_{T}} N_{x}^{2}(x, t) d x d t=I_{1}+I_{2}
$$

where

$$
\begin{aligned}
& I_{1}=\int_{0}^{T} \int_{0}^{\pi} N_{x}(x, t) w^{(1)}(x, t)\left(\int_{t}^{T} N(x, \tau) d \tau\right) d x d t, \\
& I_{2}=\int_{0}^{T} \int_{0}^{\pi} N_{x}(x, t) N(x, t)\left(\int_{t}^{T} w^{(2)}(x, \tau) d \tau\right) d x d t .
\end{aligned}
$$

We shall employ Cauchy's inequality and Wirtinger's inequality (A.1) repeatedly, as follows:

$$
I_{1} \leq\left(\int_{Q_{T}} N_{x}^{2}(x, t) d x d t\right)^{1 / 2} J_{1}
$$

where

$$
\begin{aligned}
& J_{1}^{2}:=\int_{0}^{T} \int_{0}^{\pi}\left\{\left(w^{(1)}(x, t)\right)^{2}\left(\int_{t}^{T} N(x, \tau) d \tau\right)^{2}\right\} d x d t \\
= & \int_{0}^{T} \int_{0}^{\pi}\left\{\left(\int_{0}^{x} w_{x}^{(1)}(y, t) d y\right)^{2}\left(\int_{t}^{T} N(x, \tau) d \tau\right)^{2}\right\} d x d t .
\end{aligned}
$$

Use that

$$
\begin{array}{r}
\left(\int_{0}^{x} w_{x}^{(1)}(y, t) d y\right)^{2} \leq\left(\int_{0}^{x}\left|w_{x}^{(1)}(y, t)\right| d y\right)^{2} \leq\left(\int_{0}^{\pi}\left|w_{x}^{(1)}(x, t)\right| d x\right)^{2} \\
\left(\int_{t}^{T} N(x, \tau) d \tau\right)^{2} \leq\left(\int_{t}^{T}\left(\int_{0}^{x} N_{x}(y, \tau) d y\right) d \tau\right)^{2} \leq\left(\int_{t}^{T}\left(\int_{0}^{x}\left|N_{x}(y, \tau)\right| d y\right) d \tau\right)^{2} \\
\leq\left(\int_{t}^{T}\left(\int_{0}^{\pi}\left|N_{x}(x, \tau)\right| d x\right) d \tau\right)^{2} \leq \pi T \int_{Q_{T}}\left|N_{x}(x, t)\right|^{2} d x d t
\end{array}
$$

where we have used (A.26) and (A.29). Introducing these into (A.36) we obtain

$$
\begin{aligned}
J_{1}^{2} \leq \pi T \int_{0}^{T} & \int_{0}^{\pi}\left(\int_{0}^{\pi}\left|w_{x}^{(1)}(x, t)\right| d x\right)^{2}\left(\int_{0}^{T} \int_{0}^{\pi}\left|N_{x}(x, t)\right|^{2} d x d t\right) d x d t \\
& \leq \pi T\left(\int_{Q_{T}}\left|N_{x}(x, t)\right|^{2} d x d t\right) \pi \int_{0}^{T}\left(\int_{0}^{\pi}\left|w_{x}^{(1)}(x, t)\right| d x\right)^{2} d t \\
& \leq \pi^{3} T\left(\int_{Q_{T}}\left|N_{x}(x, t)\right|^{2} d x d t\right) \int_{0}^{T}\left(\int_{0}^{\pi}\left|w_{x}^{(1)}(x, t)\right|^{2} d x\right) d t .
\end{aligned}
$$

Combining this with (A.35) we obtain

$$
\begin{aligned}
I_{1} \leq & \left(\int_{Q_{T}}\left|N_{x}(x, t)\right|^{2} d x d t\right) \pi^{3 / 2} T^{1 / 2}\left(\int_{0}^{T}\left(\int_{0}^{\pi}\left|w_{x}^{(1)}(x, t)\right|^{2} d x\right) d t\right)^{1 / 2} \\
& =\left(\int_{0}^{T}\left\|N_{x}(., t)\right\|_{L^{2}(0, \pi)}^{2} d t\right) \pi^{3 / 2} T^{1 / 2}\left(\int_{0}^{T}\left\|w_{x}^{(1)}(., t)\right\|_{L^{2}(0, \pi)}^{2} d t\right)^{1 / 2} .
\end{aligned}
$$


Similarly, we can estimate $I_{2}$ as follows. From (A.34) we have

$$
I_{2} \leq\left(\int_{Q_{T}} N_{x}^{2}(x, t) d x d t\right)^{1 / 2} J_{2}
$$

where

$$
J_{2}^{2}:=\int_{0}^{T} \int_{0}^{\pi}\left\{N^{2}(x, t)\left(\int_{t}^{T} w^{(2)}(x, \tau) d \tau\right)^{2}\right\} d x d t
$$

In (A.37), just replacing $w^{(1)}$ by $N$ and $N$ by $w^{(2)}$ we get

$$
J_{2}^{2} \leq \pi^{3} T\left(\int_{Q_{T}}\left|w_{x}^{(2)}(x, t)\right|^{2} d x d t\right) \int_{0}^{T}\left(\int_{0}^{\pi}\left|N_{x}(x, t)\right|^{2} d x\right) d t .
$$

Finally, combining (A.39) and (A.40) we obtain

$$
I_{2} \leq \pi^{3 / 2} T^{1 / 2}\left(\int_{0}^{T}\left\|N_{x}(., t)\right\|_{L^{2}(0, \pi)}^{2} d t\right)\left(\int_{0}^{T}\left\|w_{x}^{(2)}(., t)\right\|_{L^{2}(0, \pi)}^{2} d t\right)^{1 / 2} .
$$

Combining (A.38) and (A.41) in (A.32) we obtain

$$
\begin{aligned}
\int_{0}^{T}\left\|N_{x}(., t)\right\|_{L^{2}(0, \pi)}^{2} d t & \\
\leq 2 \pi^{3 / 2} T^{1 / 2} \int_{0}^{T}\left\|N_{x}(., t)\right\|_{L^{2}(0, \pi)}^{2} d t & {\left[\left(\int_{0}^{T}\left\|w_{x}^{(1)}(., t)\right\|_{L^{2}(0, \pi)}^{2} d t\right)^{1 / 2}\right.} \\
+ & \left.\left(\int_{0}^{T}\left\|w_{x}^{(2)}(., t)\right\|_{L^{2}(0, \pi)}^{2} d t\right)^{1 / 2}\right] .
\end{aligned}
$$

It remains to estimate the terms in the square bracket of (A.42).

Define

$$
\omega(x, t)=e^{\alpha t} w(x, t)
$$

where $\alpha>0$ will be prescribed later. Then (A.23), (A.25) and (A.26) become

$$
\begin{gathered}
\omega_{t}=\omega_{x x}+2\left(\int_{T}^{t} \omega(x, \tau) e^{-\alpha \tau} d \tau+\Psi^{\prime}(x)\right) \omega_{x} \\
+2\left(\int_{T}^{t} \omega_{x}(x, \tau) e^{-\alpha \tau} d \tau+\Psi^{\prime \prime}(x)\right) \omega+\alpha \omega, \\
\omega(x, T) e^{-\alpha T}-\omega(x, 0)=\theta(x), \quad x \in[0, \pi], \\
\omega(0, t)=\omega(\pi, t)=0, \quad t \in[0, T] .
\end{gathered}
$$

As in Savateev (1995), consider the sequence of linear problems

$$
\begin{aligned}
\omega_{t}^{n+1} & =\omega_{x x}^{n+1}+2\left(\int_{T}^{t} \omega^{n}(x, \tau) e^{-\alpha \tau} d \tau+\Psi^{\prime}(x)\right) \omega_{x}^{n} \\
& +2\left(\int_{T}^{t} \omega_{x}^{n}(x, \tau) e^{-\alpha \tau} d \tau+\Psi^{\prime \prime}(x)\right) \omega^{n}+\alpha \omega^{n},
\end{aligned}
$$




$$
\begin{gathered}
\omega^{n+1}(x, 0)=\omega^{n}(x, T) e^{-\alpha \tau}-\theta(x), \quad x \in[0, \pi], \\
\omega^{n+1}(0, t)=\omega^{n+1}(\pi, t)=0, \quad t \in[0, T],
\end{gathered}
$$

for $n \geq 0$, starting with $\omega^{0} \equiv 0$.

Multiply (A.46) by $\omega^{n+1}$ and integrate by parts with respect to $x$ in $L^{2}(0, \pi)$ using (A.48) to obtain

$$
\begin{aligned}
& \frac{1}{2} \frac{d}{d t}\left\|\omega^{n+1}(., t)\right\|_{L^{2}(0, \pi)}^{2}+\left\|\omega_{x}^{n+1}(., t)\right\|_{L^{2}(0, \pi)}^{2}=\alpha\left(\omega^{n}, \omega^{n+1}\right)_{L^{2}(0, \pi)} \\
& \quad-2\left(\left(\int_{T}^{t} \omega^{n}(x, \tau) e^{-\alpha \tau} d \tau+\Psi^{\prime}(x)\right) \omega^{n}, \omega_{x}^{n+1}\right)_{L^{2}(0, \pi)}, \quad n \geq 0 .
\end{aligned}
$$

In what follows, we write $\|\cdot\|_{L^{2}(0, \pi)}$ as $\|\cdot\|$.

We shall repeatedly apply the Young's inequality

$$
a b \leq \frac{a^{2}}{2 \epsilon}+\frac{\epsilon b^{2}}{2} \text { valid for any } \epsilon>0
$$

and the Wirtinger's inequality (A.1).

We estimate each term in the right hand side of (A.49). Using (A.48), (A.1) and (A.50) we get

$$
\begin{gathered}
\alpha\left(\omega^{n}, \omega^{n+1}\right) \leq \alpha^{2}\left\|\omega^{n}\right\|^{2}+\frac{1}{4}\left\|\omega^{n+1}\right\|^{2} \leq \alpha^{2}\left\|\omega_{x}^{n}\right\|^{2}+\frac{1}{4}\left\|\omega_{x}^{n+1}\right\|^{2}, \quad n \geq 0 \\
2\left(\left(\int_{T}^{t} \omega^{n}(x, \tau) e^{-\alpha \tau} d \tau\right) \omega^{n}, \omega_{x}^{n+1}\right) \\
\leq 8 \int_{0}^{\pi}\left(\omega^{n}\right)^{2}\left(\int_{T}^{t} \omega^{n} e^{-\alpha \tau} d \tau\right)^{2} d x+\frac{1}{8}\left\|\omega_{x}^{n+1}(., t)\right\|^{2} \\
=8 \int_{0}^{\pi}\left(\omega^{n}\right)^{2}\left[\int_{T}^{t} e^{-\alpha \tau}\left(\int_{0}^{x} \omega_{x}^{n}(y, \tau) d y\right) d \tau\right]^{2} d x+\frac{1}{8}\left\|\omega_{x}^{n+1}(., t)\right\|^{2} \\
\leq 8 T \pi \int_{0}^{\pi}\left(\omega^{n}(x, t)\right)^{2} d x\left(\int_{0}^{T} \int_{0}^{\pi}\left(\omega_{x}^{n}(x, t)\right)^{2} d x d t\right)+\frac{1}{8}\left\|\omega_{x}^{n+1}(., t)\right\|^{2} \\
=8 T \pi\left\|\omega_{x}^{n}(., t)\right\|^{2}\left(\int_{0}^{T}\left\|\omega_{x}^{n}(., t)\right\|^{2} d t\right)+\frac{1}{8}\left\|\omega_{x}^{n+1}(., t)\right\|^{2} .
\end{gathered}
$$

The final term in (A.49) is estimated as (use (A.1))

$$
\begin{aligned}
2\left(\Psi^{\prime}(x) \omega^{n}, \omega_{x}^{n+1}\right) & \leq 8 \int_{0}^{\pi} \Psi^{\prime 2}(x)\left(\omega^{n}(x, t)\right)^{2} d x+\frac{1}{8}\left\|\omega_{x}^{n+1}(., t)\right\|^{2} \\
& \leq 8\left(\max _{x \in[0, \pi]} \Psi^{\prime 2}(x)\right)\left\|\omega_{x}^{n}(., t)\right\|^{2}+\frac{1}{8}\left\|\omega_{x}^{n+1}(., t)\right\|^{2} .
\end{aligned}
$$

Combining (A.51)-(A.53) in (A.49) we obtain

$$
\begin{array}{r}
\frac{1}{2} \frac{d}{d t}\left\|\omega^{n+1}(., t)\right\|^{2}+ \\
\left\|\omega_{x}^{n+1}(., t)\right\|^{2} \leq\left\|\omega_{x}^{n}(., t)\right\|^{2}\left\{\alpha^{2}+8 \max _{x \in[0, \pi]} \Psi^{\prime 2}(x)\right. \\
\left.+8 T \pi\left(\int_{0}^{T}\left\|\omega_{x}^{n}(., t)\right\|^{2} d t\right)\right\}+\frac{1}{2}\left\|\omega_{x}^{n+1}(., t)\right\|^{2}
\end{array}
$$


or,

$$
\begin{array}{r}
\frac{d}{d t}\left\|\omega^{n+1}(., t)\right\|^{2}+\left\|\omega_{x}^{n+1}(., t)\right\|^{2} \leq 2\left\{\alpha^{2}+8 \max _{x \in[0, \pi]} \Psi^{\prime 2}(x)\right. \\
\left.+8 T \pi\left(\int_{0}^{T}\left\|\omega_{x}^{n}(., t)\right\|^{2} d t\right)\right\}\left\|\omega_{x}^{n}(., t)\right\|^{2}, \quad n \geq 0 .
\end{array}
$$

Integrate (A.55) with respect to $t$ from 0 to $T$ and use (A.44) and (A.45) to obtain

$$
\begin{gathered}
\left\|\omega^{n+1}(., T)\right\|^{2}+\int_{0}^{T}\left\|\omega_{x}^{n+1}(., t)\right\|^{2} d t \leq 2\|\theta\|^{2}+2 e^{-2 \alpha T}\left[\left\|\omega^{n}(., T)\right\|^{2}\right. \\
\left.+e^{2 \alpha T}\left(\alpha^{2}+8 \max _{x \in[0, \pi]} \Psi^{\prime 2}(x)\right) \int_{0}^{T}\left\|\omega_{x}^{n}(., t)\right\|^{2} d t\right]+16 T \pi\left(\int_{0}^{T}\left\|\omega_{x}^{n}(., t)\right\|^{2} d t\right)^{2} .
\end{gathered}
$$

Put

$$
y_{n}=\left\|\omega^{n}(., T)\right\|^{2}+\int_{0}^{T}\left\|\omega_{x}^{n}(., t)\right\|^{2} d t .
$$

From (A.42) and (A.57) we require

$$
4 \pi^{3 / 2} T^{1 / 2} y_{1}^{1 / 2}<1
$$

Take $b=16 \pi T, c=2\|\theta\|^{2}, a=2 e^{-2 \alpha T}$. According to the conditions of Lemma 1 we require

$$
\begin{gathered}
a=2 e^{-2 \alpha T} \in(0,1), \\
1-a=1-2 e^{-2 \alpha T} \geq \sqrt{4 b c}=8 \sqrt{2 \pi T}\|\theta\|, \\
\frac{1-a-\sqrt{(1-a)^{2}-4 b c}}{2 b} \leq y_{1} \leq \frac{1-a+\sqrt{(1-a)^{2}-4 b c}}{2 b} .
\end{gathered}
$$

Also, to satisfy (A.13), see (A.56), we observe that

$$
\begin{aligned}
& a y_{n}+b y_{n}^{2}+c=2 e^{-2 \alpha T}\left(\left\|\omega^{n}(., T)\right\|^{2}+\int_{0}^{T}\left\|\omega_{x}^{n}(., t)\right\|^{2} d t\right) \\
& +16 \pi T\left(\left\|\omega^{n}(., T)\right\|^{2}+\int_{0}^{T}\left\|\omega_{x}^{n}(., t)\right\|^{2} d t\right)^{2}+2\|\theta\|^{2} \geq y_{n+1}, \quad n \geq 0,
\end{aligned}
$$

provided that

$$
e^{2 \alpha T}\left(\alpha^{2}+8 \max _{x \in[0, \pi]} \Psi^{\prime 2}(x)\right) \leq 1 .
$$

But since $y_{0}=0$ we use (A.62) only for $n \geq 1$ and we just need to estimate $y_{1}$. For $n=0$, the system (A.46)-(A.48) gives

$$
\omega_{t}^{1}=\omega_{x x}^{1}, \quad(x, t) \in Q_{T},
$$




$$
\begin{gathered}
\omega^{1}(x, 0)=-\theta(x), \quad x \in[0, \pi], \\
\omega^{1}(0, t)=\omega^{1}(\pi, t)=0, \quad t \in[0, T] .
\end{gathered}
$$

Separation of variables gives

$$
\omega^{1}(x, t)=\sum_{n=1}^{\infty} A_{n} \sin (n x) e^{-n^{2} t}, \quad \omega_{x}^{1}(x, t)=\sum_{n=1}^{\infty} n A_{n} \cos (n x) e^{-n^{2} t},
$$

where

$$
A_{n}=-\frac{2}{\pi} \int_{0}^{T} \theta(x) \sin (n x) d x, \quad n \geq 1
$$

Then

$$
\begin{gathered}
\left\|\omega^{1}(., T)\right\|^{2}=\sum_{n=1}^{\infty} A_{n}^{2} e^{-2 n^{2} T}, \quad\left\|w_{x}^{1}(., t)\right\|^{2}=\sum_{n=1}^{\infty} n^{2} A_{n}^{2} e^{-2 n^{2} t}, \\
\int_{0}^{T}\left\|w_{x}^{1}(., t)\right\|^{2} d t=\frac{1}{2} \sum_{n=1}^{\infty} A_{n}^{2}\left(1-e^{-2 n^{2} T}\right),
\end{gathered}
$$

and

$$
y_{1}=\left\|\omega^{1}(., T)\right\|^{2}+\int_{0}^{T}\left\|\omega_{x}^{1}(., t)\right\|^{2} d t=\frac{1}{2} \sum_{n=1}^{\infty} A_{n}^{2}\left(1+e^{-2 n^{2} T}\right) .
$$

Remark that from (A.70)

$$
\frac{\|\theta\|^{2}}{2}=\frac{1}{2} \sum_{n=1}^{\infty} A_{n}^{2} \leq y_{1} \leq \sum_{n=1}^{\infty} A_{n}^{2}=\|\theta\|^{2} .
$$

Note that if $\|\theta\|=0$ then $y_{1}=0$.

Provided that there exists $\alpha>0$ satisfying (A.59)-(A.61) and (A.63) we can apply the Lemma 1 and conclude that $y_{n}$ is decreasing sequence and

$$
0 \leq y_{n} \leq y_{1}, \quad \forall n \geq 1
$$

Passing to the limit as $n \rightarrow \infty$, and using (A.42) and (A.58) are obtains that

$$
\left\|N_{x}(., t)\right\|_{L^{2}(0, \pi)}^{2}=0, \quad \forall t \in[0, T],
$$

and here $N_{x}(.,.) \equiv 0$ or using (A.30) that $N \equiv 0$. Thus the uniqueness of solution holds under the above caveat.

Remark that a necessary condition to satisfy (A.59) and (A.63) is

$$
\frac{\ln (2)}{2 T} \leq \alpha \leq \frac{1}{\sqrt{2}}
$$

